\title{
Robust nonnegative garrote variable selection in linear regression
}

\author{
I. Gijbels ${ }^{\mathrm{a}, *}$, I. Vrinssen ${ }^{\mathrm{a}}$ \\ ${ }^{a}$ KU Leuven, Department of Mathematics and Leuven Statistics Research Center (LStat), Celestijnenlaan 200B, \\ Box 2400, B-3001 Leuven, Belgium.
}

\begin{abstract}
Robust selection of variables in a linear regression model is investigated.Many variable selection methods are available, but very few methods are designed to avoid sensitivity to vertical outliers as well as to leverage points. The nonnegative garrote method is a powerful variable selection method, developed originally for linear regression but recently successfully extended to more complex regression models. The method has good performances and its theoretical properties have been established. The aim is to robustify the nonnegative garrote method for linear regression as to make it robust to vertical outliers and leverage points. Several approaches are discussed, and recommendations towards a final good performing robust nonnegative garrote method are given. The proposed method is evaluated via a simulation study that also includes a comparison with existing methods. The method performs very well, and often outperforms existing methods. A real data application illustrates the use of the method in practice.
\end{abstract}

Keywords: multiple linear regression, MM-estimation, nonnegative garrote, S-estimation, variable selection

\section{Introduction}

Variable selection has become a key issue in applied data analysis, since often many variables are measured. However, models including all the covariates are difficult to interpret and irrelevant variables increase the variance. Consider, for example, data of 60 U.S. Standard Metropolitan Statistical Areas, collected by researchers at General Motors to study whether air pollution contributes to mortality. The response is age adjusted mortality and the 14 covariates measure demographic characteristics of the cities, climate characteristics and the pollution potential of three air pollutants, namely hydrocarbon, nitrous oxide and sulfur dioxide. Our interest is to find out which of the many characteristics influence mortality and in particular, whether air pollution is significantly related to mortality. See further Section 6 .

In the literature, different variable selection methods are proposed for multiple linear regression models

$$
Y_{i}=\sum_{j=1}^{p} X_{i j} \beta_{j}+\epsilon_{i},
$$

with $\left(Y_{i}, X_{i 1}, \ldots, X_{i p}\right), i=1, \ldots, n$, i.i.d. observations from $\left(Y, X_{1}, \ldots, X_{p}\right)$, satisfying the model $Y=\sum_{j=1}^{p} X_{j} \beta_{j}+\epsilon$, where $Y$ is the response, $X_{1}, \ldots, X_{p}$ are the $p$ covariates, and $\epsilon$ is the error

\footnotetext{
*Corresponding author

Email addresses: irene.gijbels@wis.kuleuven.be; Tel.: +32 (0)16 322018 (I. Gijbels), inge.vrinssen@wis.kuleuven.be (I. Vrinssen)
} 
term with mean 0 and variance $\sigma^{2}$. We denote $\mathbf{X}_{i}=\left(X_{i 1}, \ldots, X_{i p}\right)^{\prime}$, for $i=1, \ldots, n$, with $\mathbf{A}^{\prime}$ denoting the transpose of a matrix or vector $\mathbf{A}$.

One approach for variable selection is least angle regression (LARS, Efron et al., 2004): this method sequences the candidate predictors in order of importance. Another approach is to add a penalty term $n \lambda \sum_{j=1}^{p} g\left(\beta_{j}\right)$ to the objective function of least squares regression to enforce sparsity of the model. For example, the Least Absolute Shrinkage and Selection Operator (LASSO, Tibshirani, 1996) and Bridge (Frank and Friedman, 1993; Fu, 1998) have an $L_{q}$-type of penalty on the regression coefficients, i.e. $g(\theta)=|\theta|^{q}$, with $q=1$ and $q<1$ respectively. Fan and $\mathrm{Li}(2001)$ use another penalty function, namely the Smoothly Clipped Absolute Deviation (SCAD) penalty. This penalty function $g_{\lambda}(|\cdot|)=\lambda g(|\cdot|)$ satisfies $g_{\lambda}(0)=0$ and has the first-order derivative

$$
g_{\lambda}^{\prime}(\theta)=\lambda\left\{I(\theta \leq \lambda)+\frac{(a \lambda-\theta)_{+}}{(a-1) \lambda} I(\theta>\lambda)\right\},
$$

for some $a>2$ and $\theta>0$. The nonnegative garrote (Breiman, 1995) uses a penalty on shrinkage factors of the regression coefficients. This method starts from an initial estimator, the ordinary least squares estimator (OLS), and then it shrinks or puts some coefficients of the OLS equal to zero using the nonnegative garrote shrinkage factors. Let $\widehat{\beta}_{j}^{\text {OLS }}$ denote the OLS estimator of the coefficient $\beta_{j}$, then the nonnegative garrote shrinkage factors $\widehat{\mathbf{c}}=\left(\widehat{c}_{1}, \ldots, \widehat{c}_{p}\right)^{\prime}$ are found by solving

$$
\left\{\begin{array}{l}
\widehat{\mathbf{c}}=\underset{\mathbf{c}}{\operatorname{argmin}}\left\{\frac{1}{2 n} \sum_{i=1}^{n}\left(Y_{i}-\sum_{j=1}^{p} c_{j} \widehat{\beta}_{j}^{\mathrm{OLS}} X_{i j}\right)^{2}+\lambda \sum_{j=1}^{p} c_{j}\right\} \\
\text { s.t. } 0 \leq c_{j}(j=1, \ldots, p),
\end{array}\right.
$$

for given $\lambda>0$. Breiman (1995) recommends to choose the regularization parameter $\lambda$ with five fold cross-validation. The nonnegative garrote estimator of the coefficient $\beta_{j}, j=1, \ldots, p$, is then given by

$$
\widehat{\beta}_{j}^{\mathrm{NNG}}=\widehat{c}_{j} \widehat{\beta}_{j}^{\mathrm{OLS}} .
$$

However, none of these variable selection methods are robust to outliers. Robust versions of the LARS, LASSO and SCAD methods have been considered in the literature. Khan et al. (2007) proposed a robust version of LARS (called RLARS) by replacing the mean, variance and correlation by their robust counterparts to sequence and select the important covariates. A robust regression estimator is then applied to the selected covariates. See also Agostinelli and Salibian-Barrera (2010). Different robust versions of the LASSO have been developed in the literature. See for example Owen (2006). The LAD-LASSO of Wang et al. (2007) is a penalized least absolute deviation estimator, but this method is not robust to leverage points. To overcome this drawback, Arslan (2012) proposed the WLAD-LASSO. In this method the LAD-LASSO is applied to the transformed data set $\left(w_{i} Y_{i}, w_{i} X_{i 1}, \ldots, w_{i} X_{i p}\right), i=1, \ldots, n$, where the weights $w_{i}$ are computed using robust distances. The Sparse LTS of Alfons et al. (2013) is a trimmed version of the LASSO and is also robust with respect to vertical outliers and leverage points. A robust version of the SCAD is obtained by Wang and Li (2009) and Wang et al. (2013) proposed penalized robust regression estimators based on the exponential squared loss function, where the penalty function can be of any type.

During the review process of this paper, our attention was drawn to a technical report of Medina and Ronchetti (2014). This paper deals with robust and consistent variable selection for generalized linear and additive models, using as a basis the nonnegative garrote method. In the 
framework of these models one assumes that the error distribution belongs to the exponential family. Medina and Ronchetti (2014) rely on a quasi-likelihood quantity that is robustified by using a Huber function, and by introducing a weight function (depending on the covariates) that could possibly deal with bad leverage points. The choice of the weight function needs to be made in some way; in their simulation study the authors take this function to be the identity function.

In this paper we consider a linear regression model with unspecified error distribution. Unlike the LARS, the LASSO and the Bridge, the standard nonnegative garrote method (i.e. in case a least squares estimator is used as an initial estimator) is not directly applicable to highdimensional data (i.e. the case that $p$ is much larger than $n$ ). When an initial estimator is used that can deal with the high-dimensional case, this disadvantage is also resolved. See Medina and Ronchetti (2014). The theoretical properties of the nonnegative garrotte method are well studied in the literature (Yuan and Lin, 2007) and are extended for variable selection in additive regression models and varying coefficient models by Antoniadis et al. (2012a,b). Extensive simulation studies in these papers, including comparisons with among others the LASSO and SCAD methods, revealed that the nonnegative garrote method overall performs quite well. We therefore, in this paper, robustify the nonnegative garrote for outliers in the response and in the covariates by using robust alternatives to the least squares regression estimator, such as the S-estimator (Rousseeuw and Yohai, 1984) and the least trimmed squares (LTS) estimator (Rousseeuw, 1984). We also develop a reweighting step that is related with the MM-estimator of Yohai (1987), to increase the efficiency of the proposed robust nonnegative garrote method under the normal error model. Our study indicates that a carefully designed robust nonnegative garrote method performs quite well and often outperforms other available methods. This paper thus contributes in a thorough study of the different approaches to robustify the nonnegative garrote method (on all levels of the estimation procedure) for linear regression models.

The rest of the paper is organized as follows. In Section 2, we present three robust versions of the nonnegative garrote, namely the M-, S- and LTS-nonnegative garrote. In Section 3, we give a first simulation study to compare these proposed methods. In Section 4 an extra reweighting step is proposed to improve the results of the S-nonnegative garrote. Section 5 presents then a more extensive simulation study to compare the performances of the robust nonnegative garrote methods with other robust variable selection methods. In Section 6 a real-data application is given and Section 7 provides some further discussions and concludes. The proofs of the theorems can be found in the Appendix.

\section{Robust nonnegative garrote method}

The original nonnegative garrote consists of three parts, namely the initial estimation of $\boldsymbol{\beta}=$ $\left(\beta_{1}, \ldots, \beta_{p}\right)^{\prime}$, the estimation of the nonnegative shrinkage factors $\mathbf{c}=\left(c_{1}, \ldots, c_{p}\right)^{\prime}$ and the selection of the regularization parameter $\lambda$. For these three parts we need to find appropriate robust alternatives, as will be discussed in the subsequent Sections 2.1, 2.2, and 2.3.

\subsection{Robust initial estimator}

Several robust alternatives to ordinary least squares regression are available, such as the Mestimator (Huber, 1973), the S-estimator, the MM-estimator, the $\tau$-estimator (Yohai and Zamar, 1988 ) and the LTS-estimator. A general overview of these methods can be found in Maronna et al. (2006). See also Heritier et al. (2009). Since these robust alternatives can be used as the initial estimator of the coefficients $\boldsymbol{\beta}$, we will briefly discuss them. 
The M-estimation approach, to make the ordinary least squares regression more robust to vertical outliers, consists of replacing the quadratic loss function by a different slowly increasing function $\rho$ which is symmetric with a unique minimum at zero. To obtain a scale-invariant estimator, the residuals need to be standardized by a robust estimate of their scale $\widehat{\sigma}$. The M-estimator is defined as

$$
\widehat{\boldsymbol{\beta}}=\underset{\boldsymbol{\beta}}{\operatorname{argmin}} \sum_{i=1}^{n} \rho\left(\frac{Y_{i}-\mathbf{X}_{i}^{\prime} \boldsymbol{\beta}}{\widehat{\sigma}}\right) .
$$

A frequently used loss function is for example a loss function of Tukey's biweight family,

$$
\rho_{d}(x)=\left\{\begin{array}{cl}
1-\left(1-\left(\frac{x}{d}\right)^{2}\right)^{3} & \text { if }|x| \leq d, \\
1 & \text { if }|x|>d
\end{array}\right.
$$

where the constant $d$ can be tuned for estimation efficiency.

Since the ordinary least squares estimators are the coefficients $\boldsymbol{\beta}$ that produce residuals with minimal scale, the $\tau$-estimator looks for coefficients that produce residuals that minimize a robust scale estimator of the residuals, such as the $\tau$-scale.

Take two loss function $\rho_{0}$ and $\rho_{1}$ that satisfy Assumption (A):

(A). Let $\rho$ be a real function such that:

1. $\rho$ is symmetric, continuously differentiable and $\rho(0)=0$.

2. There exists $d>0$ such that $\rho$ is strictly increasing on $[0, d]$ and constant on $[d, \infty)$ with $0<\rho(d)=a<+\infty$.

Denote the constants $E\left(\rho_{0}(Z)\right)$ and $E\left(\rho_{1}(Z)\right)$, with $Z$ standard normal distributed, by $b_{0}$ and $b_{1}$ respectively. Given the residuals $\mathbf{r}(\boldsymbol{\beta})=\left(r_{1}, \ldots, r_{n}\right)^{\prime}$ with $r_{i}=Y_{i}-\mathbf{X}_{i}^{\prime} \boldsymbol{\beta}, i=1, \ldots, n$, let $\widehat{\sigma}(\mathbf{r}(\boldsymbol{\beta}))$ be an M-scale that solves

$$
\frac{1}{n} \sum_{i=1}^{n} \rho_{0}\left(\frac{Y_{i}-\mathbf{X}_{i}^{\prime} \boldsymbol{\beta}}{\widehat{\sigma}(\mathbf{r}(\boldsymbol{\beta}))}\right)=b_{0} .
$$

The $\tau$-scale estimator $\tau(\mathbf{r}(\boldsymbol{\beta}))$ is then defined as

$$
\tau^{2}(\mathbf{r}(\boldsymbol{\beta}))=\widehat{\sigma}^{2}(\mathbf{r}(\boldsymbol{\beta})) \frac{1}{n b_{1}} \sum_{i=1}^{n} \rho_{1}\left(\frac{Y_{i}-\sum_{j=1}^{p} \beta_{j} X_{i j}}{\widehat{\sigma}(\mathbf{r}(\boldsymbol{\beta}))}\right),
$$

and the $\tau$-estimator is given by

$$
\widehat{\boldsymbol{\beta}}=\underset{\boldsymbol{\beta}}{\operatorname{argmin}} \tau(\mathbf{r}(\boldsymbol{\beta})) .
$$

If $\rho_{0}=\rho_{1}$, the $\tau$-scale is reduced to the M-scale and the solution of the resulting optimization problem is called the S-estimator.

The MM-estimator applies an extra reweighting step on a robust estimator for the coefficients $\boldsymbol{\beta}$ to increase the efficiency.

Consider two loss functions $\rho_{0}$ and $\rho_{1}$ that satisfy Assumption (A) and for which $\rho_{1}(u) \leq$ $\rho_{0}(u), \forall u \in \mathbb{R}, \sup _{x \in \mathbb{R}} \rho_{0}(x)=\sup _{x \in \mathbb{R}} \rho_{1}(x)$ and $E\left(\rho_{0}(Z)\right)=0.5$, with $Z$ a standard normal distributed variable. The MM-estimator is obtained in three steps: 
1. Compute an initial consistent estimate of the coefficients $\boldsymbol{\beta}$, denoted by $\widehat{\boldsymbol{\beta}}^{0}$, with high breakdown point, but possibly low efficiency.

2. Compute the M-scale of the residuals $\mathbf{r}\left(\widehat{\boldsymbol{\beta}}^{0}\right)$, denoted by $\widehat{\sigma}\left(\mathbf{r}\left(\widehat{\boldsymbol{\beta}}^{0}\right)\right)$, that solves (3) using the function $\rho_{0}$.

3. The MM-estimator is then found by solving (2) with loss function $\rho_{1}$ and scale $\widehat{\sigma}\left(\mathbf{r}\left(\widehat{\boldsymbol{\beta}}^{0}\right)\right)$.

Least trimmed squares estimation (LTS) accords to finding the subset of $h \leq n$ observations whose least squares fit realizes residuals with the smallest scale. Hence, the LTS-estimator is defined as

$$
\widehat{\boldsymbol{\beta}}=\underset{\boldsymbol{\beta}}{\operatorname{argmin}} \sum_{i=1}^{h}\left(\mathbf{r}^{2}(\boldsymbol{\beta})\right)_{i: n},
$$

where $\left(\mathbf{r}^{2}(\boldsymbol{\beta})\right)_{1: n} \leq \cdots \leq\left(\mathbf{r}^{2}(\boldsymbol{\beta})\right)_{n: n}$ are the order statistics of the squared residuals.

Table 1: Summary robust estimators

\begin{tabular}{lccc}
\hline Method & $\begin{array}{c}\text { Robust to } \\
\text { vertical outliers? }\end{array}$ & $\begin{array}{c}\text { Robust to } \\
\text { leverage points? }\end{array}$ & Efficiency? \\
\hline OLS & no & no & high \\
M & yes & no & high \\
$\tau$ & yes & yes & high \\
S & yes & yes & low \\
MM & yes & yes & high \\
LTS & yes & yes & low \\
\hline
\end{tabular}

A summary of the robust estimation methods discussed in this section, can be found in Table 1 . In this table one can find whether a method is robust to vertical outliers and/or leverage points and whether the efficiency of the method is either high or low.

In general, it is better to use a method that is robust to vertical outliers and leverage points, and that is highly efficient. The simulation study of Section 3 shows that this is also the case for the initial estimation of the coefficients $\boldsymbol{\beta}$. Thus, extrapolating this idea to the estimation of the nonnegative garrote shrinkage factors gives that we want to develop a method based on the MM-estimator or the $\tau$-estimator. However, we will not use the $\tau$-estimator to robustify the estimation method of the nonnegative garrote shrinkage factors, since its definition is rather complex. Therefore, we first propose estimation methods for the nonnegative garrote shrinkage factors based on the S-estimator and the LTS-estimator. The outcomes of these methods can then be used as a starting point for the nonnegative garrote shrinkage factors estimation method based on the MM-estimator.

\subsection{Robust nonnegative garrote methods}

We will propose three robust methods to estimate the nonnegative garrote shrinkage factors. These optimization problems are based on the S, M and LTS-estimator. Suppose in the following that we already found a robust initial estimator for the coefficient $\beta_{j}, j=1, \ldots, p$, and denote this estimator by $\widehat{\beta}_{j}^{\mathrm{R}}, j=1, \ldots, p$. Then let $Z_{i j}^{\mathrm{R}}=\widehat{\beta}_{j}^{\mathrm{R}} X_{i j}$ for $j=1, \ldots, p, i=1, \ldots, n$, such that $\boldsymbol{Z}^{\mathrm{R}}=\left(Z_{i j}^{\mathrm{R}}\right)_{1 \leq i \leq n, 1 \leq j \leq p}$ and $\boldsymbol{Z}_{i}^{\mathrm{R}}=\left(Z_{i 1}^{\mathrm{R}}, \ldots Z_{i p}^{\mathrm{R}}\right)^{\prime}, i=1, \ldots, n$. Also denote the vector of 
responses by $\mathbf{Y}=\left(Y_{1}, Y_{2}, \ldots, Y_{n}\right)^{\prime}$, the residuals of the robust nonnegative garrote shrinkage factors $\mathbf{c}$ with $\mathbf{r}_{\mathrm{R}}(\mathbf{c})=\left(r_{\mathrm{R}, 1}, \ldots, r_{\mathrm{R}, n}\right)^{\prime}$, where $r_{\mathrm{R}, i}=Y_{i}-\sum_{j=1}^{p} c_{j} Z_{i j}^{\mathrm{R}}, i=1, \ldots, n$, and the vector of ones of length $p$ by $\mathbf{1}_{p}=(1,1, \ldots, 1)^{\prime}$. Robust nonnegative garrote shrinkage factors $\widehat{\mathbf{c}}$ are found by solving the optimization problem

$$
\left\{\begin{array}{l}
\widehat{\mathbf{c}}=\underset{\mathbf{c}}{\operatorname{argmin}}\left\{\operatorname{GOF}\left(\mathbf{r}_{\mathrm{R}}(\mathbf{c})\right)+\lambda \sum_{j=1}^{p} c_{j}\right\} \\
\text { s.t. } c_{j} \geq 0(j=1, \ldots, p),
\end{array}\right.
$$

where $\operatorname{GOF}\left(\mathbf{r}_{\mathrm{R}}(\mathbf{c})\right)$ is a robust alternative to the mean squared residuals in (1). In particular,

- $\operatorname{GOF}\left(\mathbf{r}_{\mathrm{R}}(\mathbf{c})\right)=\frac{1}{n} \sum_{i=1}^{n} \rho\left(\frac{Y_{i}-\sum_{j=1}^{p} c_{j} Z_{i j}^{\mathrm{R}}}{\widehat{\sigma}}\right)$, where $\widehat{\sigma}$ is a robust scale estimator of the residuals $\mathbf{r}\left(\widehat{\boldsymbol{\beta}}^{\mathrm{R}}\right)$ of the initial estimators of the coefficients $\boldsymbol{\beta}$ and $\rho$ is a nonnegative, symmetric and slowly increasing function with a unique minimum at zero, leads to the M-nonnegative garrote method;

- $\operatorname{GOF}\left(\mathbf{r}_{\mathrm{R}}(\mathbf{c})\right)=\widehat{\sigma}\left(\mathbf{r}_{\mathrm{R}}(\mathbf{c})\right)$ where $\widehat{\sigma}\left(\mathbf{r}_{\mathrm{R}}(\mathbf{c})\right)$ solves

$$
\frac{1}{n} \sum_{i=1}^{n} \rho\left(\frac{Y_{i}-\sum_{j=1}^{p} c_{j} Z_{i j}^{\mathrm{R}}}{\widehat{\sigma}\left(\mathbf{r}_{\mathrm{R}}(\mathbf{c})\right)}\right)=b,
$$

where $\rho$ is a loss function of Tukey's biweight family with $d=1.547$ and $b=0.5$, gives the S-nonnegative garrote method;

- $\operatorname{GOF}\left(\mathbf{r}_{\mathrm{R}}(\mathbf{c})\right)=\frac{1}{2 h} \sum_{i=1}^{h}\left(\mathbf{r}_{\mathrm{R}}^{2}(\mathbf{c})\right)_{i: n}$, with $h \leq n$, results in the LTS-nonnegative garrote method. If $h=n$ this coincides with dealing with the objective function in (1) of the original nonnegative garrote method.

To simplify the notation we will use $\boldsymbol{Z}$ for $\boldsymbol{Z}^{\mathrm{R}}$ and $\boldsymbol{Z}_{i}$ for $\boldsymbol{Z}_{i}^{\mathrm{R}}$ in the sequel. The next results show that the optimization problems of the M- and S-nonnegative garrote method can be written as weighted quadratic programming problems. These weighted quadratic programming problems suggest iterative procedures to compute the M- and S-nonnegative garrote shrinkage factors. The proofs of Theorems 2.1 and 2.2 can be found in the Appendix.

Theorem 2.1. Let $\mathbf{c}^{0}$ be the current value of $\mathbf{c}$ in the iteration procedure. Then, the optimization problem of the M-nonnegative garrote method in the next iteration step can be written as

$$
\left\{\begin{array}{l}
\widehat{\mathbf{c}}=\underset{\mathbf{c}}{\operatorname{argmin}}\left\{\frac{1}{2} \mathbf{c}^{\prime} \boldsymbol{Z}^{\prime} \mathbf{W}_{M}\left(\widehat{\mathbf{c}}^{0}\right) \boldsymbol{Z} \mathbf{c}-\left(\boldsymbol{Z}^{\prime} \mathbf{W}_{M}\left(\widehat{\mathbf{c}}^{0}\right) \mathbf{Y}-n \widehat{\sigma}^{2} \lambda \mathbf{1}_{p}\right)^{\prime} \mathbf{c}\right\} \\
\text { s.t. } c_{j} \geq 0(j=1, \ldots, p),
\end{array}\right.
$$

where $\mathbf{W}_{M}(\mathbf{c})=\operatorname{diag}\left(W_{M, i}(\mathbf{c})\right) \in \mathbb{R}^{n \times n}$ with $W_{M, i}(\mathbf{c})=\rho^{\prime}\left(\frac{r_{\mathrm{R}, i}(\mathbf{c})}{\widehat{\sigma}}\right)\left(\frac{\widehat{\sigma}}{r_{\mathrm{R}, i}(\mathbf{c})}\right)$.

Theorem 2.2. Let $\mathbf{c}^{0}$ be the current value of $\mathbf{c}$ in the iteration procedure. Then, the optimization problem of the S-nonnegative garrote method in the next iteration step can be written as

$$
\left\{\begin{array}{l}
\widehat{\mathbf{c}}=\underset{\mathbf{c}}{\operatorname{argmin}}\left\{\frac{1}{2} \mathbf{c}^{\prime} \boldsymbol{Z}^{\prime} \mathbf{W}_{S}\left(\widehat{\mathbf{c}}^{0}\right) \boldsymbol{Z} \mathbf{c}-\left(\boldsymbol{Z}^{\prime} \mathbf{W}_{S}\left(\widehat{\mathbf{c}}^{0}\right) \mathbf{Y}-\frac{\lambda}{\omega_{S}(\widehat{\mathbf{c}})} \mathbf{1}_{p}\right)^{\prime} \mathbf{c}\right\} \\
\text { s.t. } c_{j} \geq 0(j=1, \ldots, p),
\end{array}\right.
$$


where $\mathbf{W}_{S}(\mathbf{c})=\operatorname{diag}\left(W_{S, i}(\mathbf{c})\right) \in \mathbb{R}^{n \times n}$ with $W_{S, i}(\mathbf{c})=\rho^{\prime}\left(\frac{r_{\mathrm{R}, i}(\mathbf{c})}{\widehat{\sigma}\left(\mathbf{r}_{\mathrm{R}}(\mathbf{c})\right)}\right)\left(\frac{\widehat{\sigma}\left(\mathbf{r}_{\mathrm{R}}(\mathbf{c})\right)}{r_{\mathrm{R}, i}(\mathbf{c})}\right), \omega_{S}(\mathbf{c})=$ $\frac{\widehat{\sigma}\left(\mathbf{r}_{\mathrm{R}}(\mathbf{c})\right)}{\mathbf{r}_{\mathrm{R}}^{\prime}(\mathbf{c}) \mathbf{W}_{S}(\mathbf{c}) \mathbf{r}_{\mathrm{R}}(\mathbf{c})}$ and $\widehat{\sigma}\left(\mathbf{r}_{\mathrm{R}}(\mathbf{c})\right)$ solves $(5)$.

We next discuss (fast) algorithms for practical implementation of the iterative procedures.

\section{Algorithms}

First, a procedure to compute the M-nonnegative garrote shrinkage factors is given. Note that the weights in the objective function of (6) depend on $\widehat{\mathbf{c}}^{0}$. Optimization problems of the form

$$
\left\{\begin{array}{l}
\widehat{\mathbf{c}}_{k+1}=\underset{\mathbf{c}_{k+1}}{\operatorname{argmin}}\left\{\frac{1}{2} \mathbf{c}_{k+1}^{\prime} \boldsymbol{Z}^{\prime} \mathbf{W}_{M}\left(\widehat{\mathbf{c}}_{k}\right) \boldsymbol{Z} \mathbf{c}_{k+1}-\left(\boldsymbol{Z}^{\prime} \mathbf{W}_{M}\left(\widehat{\mathbf{c}}_{k}\right) \mathbf{Y}-n \widehat{\sigma}^{2} \lambda \mathbf{1}_{p}\right)^{\prime} \mathbf{c}_{k+1}\right\} \\
\text { s.t. } c_{k+1, j} \geq 0(j=1, \ldots, p),
\end{array}\right.
$$

for $k=0,1,2, \ldots$, need to be solved iteratively to find a critical point of (4a) for the Mnonnegative garrote method. These iterations are performed until convergence and the resulting critical point $\widehat{\mathbf{c}}$ is then the final estimate of the M-nonnegative garrote shrinkage factors $\mathbf{c}$. The solution of the following quadratic programming problem

$$
\left\{\begin{array}{l}
\widehat{\mathbf{c}}^{0}=\underset{\mathbf{c}}{\operatorname{argmin}}\left\{\frac{1}{2} \mathbf{c}^{\prime} \boldsymbol{Z}^{\prime} \boldsymbol{Z} \mathbf{c}-\left(\boldsymbol{Z}^{\prime} \mathbf{Y}-n \widehat{\sigma}^{2} \lambda \mathbf{1}_{p}\right)^{\prime} \mathbf{c}\right\} \\
\text { s.t. } c_{j} \geq 0(j=1, \ldots, p),
\end{array}\right.
$$

can be used as a starting value for the iterative procedure. This quadratic programming problem is analogue to (6), but the squared loss function is used for the nonnegative loss function $\rho$ in the weight matrix $\mathbf{W}_{M}(\widehat{\mathbf{c}})$.

We will adapt the fast algorithm for S-regression estimates of Salibian-Barrera and Yohai (2006) and the FAST-LTS algorithm of Rousseeuw and Driessen (2006) to get algorithms to compute the S- and LTS-nonnegative garrote shrinkage factors. The structure of these algorithms is the same, but the algorithm for the S-nonnegative garrote shrinkage factors is based on local improvement steps or I-steps, while the algorithm for the LTS-nonnegative garrote shrinkage factors is based on concentrations steps or C-steps.

In an I-step one step of the iterative procedure for solving optimization problem (7) is performed. Hence, if $\widehat{\mathbf{c}}_{k}$ is an approximation of $\mathbf{c}$, this I-step is defined as follows:

1. Compute $\widehat{\sigma}_{k}=\widehat{\sigma}\left(\mathbf{r}_{\mathrm{R}}\left(\widehat{\mathbf{c}}_{k}\right)\right)$, the weight matrix $\mathbf{W}_{k}=\operatorname{diag}\left(W_{S, i}\left(\widehat{\mathbf{c}}_{k}\right)\right) \in \mathbb{R}^{n \times n}$ with weights $W_{S, i}\left(\widehat{\mathbf{c}}_{k}\right)=\rho^{\prime}\left(r_{\mathrm{R}, i}\left(\widehat{\mathbf{c}}_{k}\right) / \widehat{\sigma}_{k}\right)\left(\widehat{\sigma}_{k} / r_{\mathrm{R}, i}\left(\widehat{\mathbf{c}}_{k}\right)\right)$ and $\omega_{k}=\widehat{\sigma}_{k} /\left(\mathbf{r}_{\mathrm{R}}^{\prime}\left(\widehat{\mathbf{c}}_{k}\right) \mathbf{W}_{k} \mathbf{r}_{\mathrm{R}}\left(\widehat{\mathbf{c}}_{k}\right)\right)$.

2. Solve the quadratic programming problem

$$
\left\{\begin{array}{l}
\widehat{\mathbf{c}}_{k+1}=\underset{\mathbf{c}}{\operatorname{argmin}}\left\{\frac{1}{2} \mathbf{c}^{\prime} \boldsymbol{Z}^{\prime} \mathbf{W}_{k} \boldsymbol{Z} \mathbf{c}-\left(\boldsymbol{Z}^{\prime} \mathbf{W}_{k} \mathbf{Y}-\frac{\lambda}{\omega_{k}} \mathbf{1}_{p}\right)^{\prime} \mathbf{c}\right\} \\
\text { s.t. } c_{j} \geq 0(j=1, \ldots, p),
\end{array}\right.
$$

to find a better approximation of $\mathbf{c}$.

Since the LTS-nonnegative garrote corresponds to finding the subset of $h \leq n$ residual observations with the smallest penalized residual sum of squares, the C-step works as follows. Suppose that $\widehat{\mathbf{c}}_{k}$ are the estimates of the nonnegative garrote shrinkage factors based on a subset $H_{k} \subset\{1, \ldots, n\}$ with $\left|H_{k}\right|=h$. Construct the next subset $H_{k+1}$ from the observations with the $h$ smallest absolute values of the residuals $\mathbf{r}_{k}=\mathbf{r}_{\mathrm{R}}\left(\widehat{\mathbf{c}}_{k}\right)$,

$$
H_{k+1}=\left\{i \in\{1, \ldots, n\}:\left|r_{k, i}\right| \in\left\{\left|\mathbf{r}_{k}\right|_{j: n}: j=1, \ldots, h\right\}\right\}
$$


and compute $\widehat{\mathbf{c}}_{k+1}$, the estimates of the nonnegative garrote shrinkage factors based on $H_{k+1}$. Let $Q_{k}=\sum_{i \in H_{k}} \mathbf{r}_{\mathrm{R}, i}^{2}\left(\widehat{\mathbf{c}}_{k}\right)+h \lambda \sum_{j=1}^{p} \widehat{c}_{k, j}$, then it holds that $Q_{k+1} \leq Q_{k}$.

This is valid, since $\sum_{i \in H_{k+1}} \mathbf{r}_{\mathrm{R}, i}^{2}\left(\widehat{\mathbf{c}}_{k}\right)+h \lambda \sum_{j=1}^{p} \widehat{c}_{k, j} \leq Q_{k}$. Further, the estimates of the nonnegative garrote shrinkage factors $\widehat{\mathbf{c}}_{k+1}$ of these $h$ observations minimize $Q_{k+1}$ so that $Q_{k+1} \leq \sum_{i \in H_{k+1}} \mathbf{r}_{\mathrm{R}, i}^{2}\left(\widehat{\mathbf{c}}_{k}\right)+h \lambda \sum_{j=1}^{p} \widehat{c}_{k, j} \leq Q_{k}$.

Since the objective functions (4a) for the S- and LTS-nonnegative garrote are in general nonconvex, different starting points may converge to different critical points. A large number $N$ of starting values is therefore needed. To find a starting point $\mathbf{c}^{(0)}$ for the S-nonnegative garrote, draw a random subsample of size $p$ of the data set, denote the subsample by $\left(\mathbf{Y}_{*}, \boldsymbol{Z}_{*}\right)$, and let $\mathbf{c}^{(0)}$ be the solution of the quadratic programming problem

$$
\left\{\begin{array}{l}
\mathbf{c}^{(0)}=\underset{\mathbf{c}}{\operatorname{argmin}}\left\{\frac{1}{2} \mathbf{c}^{\prime} \boldsymbol{Z}_{*}^{\prime} \boldsymbol{Z}_{*} \mathbf{c}-\left(\boldsymbol{Z}_{*}^{\prime} \mathbf{Y}_{*}-\lambda \mathbf{1}_{p}\right)^{\prime} \mathbf{c}\right\} \\
\text { s.t. } c_{j} \geq 0(j=1, \ldots, p) .
\end{array}\right.
$$

For a recent contribution to subsampling for S-estimators, see Koller (2012).

There are several approaches to find a starting point $\mathbf{c}^{(0)}$ for the LTS-nonnegative garrote. One approach is to select $h$ data points at random and compute the estimates of the nonnegative garrote shrinkage factors based on this sample. Another approach is the following. First randomly select a subsample of size $p$ of the data set and denote this subsample by $\left(\mathbf{Y}_{*}, \boldsymbol{Z}_{*}\right)$. Let $\widehat{\mathbf{c}}_{*}$ be the solution of the quadratic programming problem

$$
\left\{\begin{array}{l}
\widehat{\mathbf{c}}_{*}=\underset{\mathbf{c}}{\operatorname{argmin}}\left\{\frac{1}{2} \mathbf{c}^{\prime} \boldsymbol{Z}_{*}^{\prime} \boldsymbol{Z}_{*} \mathbf{c}-\left(\boldsymbol{Z}_{*}^{\prime} \mathbf{Y}_{*}-p \lambda \mathbf{1}_{p}\right)^{\prime} \mathbf{c}\right\} \\
\text { s.t. } c_{j} \geq 0(j=1, \ldots, p),
\end{array}\right.
$$

and compute now the residuals $\mathbf{r}_{*}=\mathbf{r}_{\mathrm{R}}\left(\widehat{\mathbf{c}}_{*}\right)$. The initial subsample $H_{0}$ is then given by the indices of the $h$ observations with the smallest absolute values of the residuals $\mathbf{r}_{*}$ and $\mathbf{c}^{(0)}$ is the estimates of the nonnegative garrote shrinkage factors based on $H_{0}$. Since the probability of having a subset of size $p$ without outliers is higher than for a subset of size $h$, the second approach returns more good initial subsamples than the first approach. Therefore, we will use the second approach.

The following procedure can now be used to compute the S- and LTS-nonnegative garrote shrinkage factors:

1. Let $\mathbf{c}_{1}^{(0)}, \ldots, \mathbf{c}_{N}^{(0)}$ be initial candidates. For each $\mathbf{c}_{\ell}^{(0)}$,

(a) Carry out $k$ I-steps or C-steps and denote the improved candidate with $\mathbf{c}_{\ell}^{(1)}$.

(b) Only for the S-nonnegative garrote method: compute the M-scale $\widehat{\sigma}_{\ell}=\widehat{\sigma}\left(\mathbf{r}_{\mathrm{R}}\left(\mathbf{c}_{\ell}^{(1)}\right)\right)$.

2. Keep the $t$ improved candidates with the lowest values of the objective function (4a).

3. For each $\mathbf{c}_{\ell}^{(1)}, \ell=1, \ldots, t$, carry out I-steps or C-steps until convergence and denote the final candidate with $\mathbf{c}_{\ell}^{F}$.

4. The estimates of the S- or LTS-nonnegative garrote shrinkage factors is the candidate $\mathbf{c}_{\ell}^{F}$ with the lowest value of (4a).

Frequently used values for $N, k$ and $t$ for, respectively, the S- and LTS-nonnegative garrote method are, respectively, $N=500, k=2$ and $t=5$ and $N=500, k=2$ and $t=10$. 


\subsection{Robust selection of regularization parameter $\lambda$}

Since all discussed optimization problems depend on the regularization parameter $\lambda$, procedures for selecting this parameter are necessary. In the simulation studies, several robust data-driven criteria for choosing $\lambda$ are compared. Possible procedures are the selection of $\lambda$ that minimizes robust versions of Akaike's Information Criterion, of the Bayesian Information Criterion or of the repeated five fold cross-validation; or the use of a robust version of the L-curve criterion.

The robust Akaike's Information Criterion (RAIC) for the S- or LTS-nonnegative garrote is given by

$$
\mathrm{RAIC}=\log \left(\widehat{\sigma}\left(\mathbf{r}_{\mathrm{R}}(\widehat{\mathbf{c}})\right)\right)+\frac{2}{n} \mathrm{df}
$$

and the robust Bayesian Information Criterion (RBIC) for the S- or LTS-nonnegative garrote is defined as

$$
\mathrm{RBIC}=\log \left(\widehat{\sigma}\left(\mathbf{r}_{\mathrm{R}}(\widehat{\mathbf{c}})\right)\right)+\frac{\log (n)}{n} \mathrm{df},
$$

where the degrees of freedom (df) are estimated by the number of nonzero S- or LTS-nonnegative garrote estimates and $\widehat{\sigma}\left(\mathbf{r}_{\mathrm{R}}(\widehat{\mathbf{c}})\right)$ is the M-scale of the residuals of the S- or LTS-nonnegative garrote shrinkage factors. The RAIC and RBIC for the M-nonnegative garrote are obtained by replacing $\widehat{\sigma}\left(\mathbf{r}_{\mathrm{R}}(\widehat{\mathbf{c}})\right)$ with $\frac{1}{n} \sum_{i=1}^{n} \rho\left(\frac{Y_{i}-\boldsymbol{Z}_{i}^{\prime} \widehat{\mathbf{c}}}{\widehat{\sigma}}\right)$.

To select the regularization parameter with robust five fold cross-validation, the data set is randomly divided in five blocks of approximately equal size. The training sample then consists of four blocks and the validation sample is given by the left-out block. Because each block is left out once, a prediction for each observation in the data set is obtained. The average prediction error is then calculated based on $M$ possible random five fold splits of the sample. Hence, the robust five fold cross-validation (RCV) is given by

$$
\mathrm{RCV}=\frac{1}{M} \sum_{m=1}^{M} g\left(Y_{i}-\boldsymbol{Z}_{i}^{\prime} \widehat{\mathbf{c}}^{-i_{m}}\right),
$$

where $g\left(Y_{i}-\boldsymbol{Z}_{i}^{\prime} \widehat{\mathbf{c}}^{-i_{m}}\right)$ is a robust alternative for the mean squared error, for example the trimmed mean squared error. For each observation $i, \widehat{\mathbf{c}}^{-i_{m}}$ denotes the estimate of $\mathbf{c}$ obtained with the S-, LTS- or M-nonnegative garrote, but without using the block containing observation $i$ in the $m$ th random run of five fold cross-validation.

Since it is very time-consuming to compute the estimates $\widehat{\mathbf{c}}^{-i_{m}} 5 M$ times exactly, we want to speed up this algorithm (Khan et al., 2010). Therefore, these estimates are approximated by first computing, for example, the S-nonnegative garrote shrinkage factors $\widehat{\mathbf{c}}$ exactly for the complete data set. With the estimate of scale $\widehat{\sigma}\left(\mathbf{r}_{\mathrm{R}}(\widehat{\mathbf{c}})\right)$, the estimated weights $\mathbf{W}_{S}(\widehat{\mathbf{c}})$ and $\omega_{S}(\widehat{\mathbf{c}})$ from the complete data set, an initial approximation of the estimate $\widehat{\mathbf{c}}$ for the training sample $\left(\mathbf{Y}^{*}, \boldsymbol{Z}^{*}\right)$ can be found by solving

$$
\left\{\begin{array}{l}
\widehat{\mathbf{c}}_{0}=\underset{\mathbf{c}}{\operatorname{argmin}}\left\{\frac{1}{2} \mathbf{c}^{\prime} \boldsymbol{Z}^{* \prime} \mathbf{W}_{S}(\widehat{\mathbf{c}}) \boldsymbol{Z}^{*} \mathbf{c}-\left(\boldsymbol{Z}^{* \prime} \mathbf{W}_{S}(\widehat{\mathbf{c}}) \mathbf{Y}^{*}-\frac{\lambda}{\omega_{S}(\widehat{\mathbf{c}})} \mathbf{1}_{p}\right)^{\prime} \mathbf{c}\right\} \\
\text { s.t. } c_{j} \geq 0(j=1, \ldots, p) .
\end{array}\right.
$$

This initial approximation can now be improved by applying 2 I-steps. The approximation of $\widehat{\mathbf{c}}^{-i_{m}}$ for the M- and LTS-nonnegative garrote is done in a similar way.

The last data-driven selection criterion that we consider, is a robust version of the L-curve criterion (see for example Belge et al., 2002). This criterion is based on the L-curve, which 
plots the rescaled fitted quantities against the rescaled penalty terms and then searches for the point with the smallest distance to the origin. The fitted quantities and the penalty terms are rescaled, so that their inputs have the range $(0,1)$. The scaling is done with the following formula, for a vector $\mathbf{x},(\mathbf{x}-\min (\mathbf{x})) /(\max (\mathbf{x})-\min (\mathbf{x}))$. The fitted quantity of the S-, M- and LTS-nonnegative garrote are $\widehat{\sigma}\left(\mathbf{r}_{\mathrm{R}}(\widehat{\mathbf{c}})\right), \frac{1}{n} \sum_{i=1}^{n} \rho\left(\frac{Y_{i}-\boldsymbol{Z}_{i}^{\prime} \widehat{\mathbf{c}}}{\widehat{\sigma}}\right)$ and $\frac{1}{h} \sum_{i=1}^{h}\left(\mathbf{r}_{\mathrm{R}}^{2}(\widehat{\mathbf{c}})\right)_{i: n}$ respectively.

\section{Simulation study: part I}

The goal of this simulation study is to investigate the finite-sample performances of the various robustifications of the nonnegative garrote variable selection method. Based on this study we then proceed by a further improvement, in Section 4. Section 5 is then devoted to a second part of the simulation study in which we compare the performance of the recommended robust nonnegative garrote method with that of other methods.

In this first part we consider a simulation model with 10 covariates and no intercept, given by

$$
Y_{i}=X_{i}^{\prime} \boldsymbol{\beta}+\sigma \epsilon_{i}=3 X_{i 1}+1.5 X_{i 2}+2 X_{i 5}+2 X_{i 6}+X_{i 10}+\sigma \epsilon_{i}
$$

where the covariates $X_{i 1}, \ldots, X_{i 10}$ are generated from a multivariate normal distribution with mean 0 and $\operatorname{Corr}\left(X_{i j}, X_{i k}\right)=0.5^{|j-k|}$ for $j, k=1, \ldots, 10$. The error term $\epsilon_{i}$ is standard normal distributed and $\sigma=3$, so that the signal-to-noise ratio is $\frac{\operatorname{Var}\left(X^{\prime} \beta\right)}{\sigma^{2}}=\frac{4019}{1152} \approx 3.49$. To investigate the performances of the different methods, we consider three different contamination schemes:

1. No contamination ("no").

2. Vertical outliers ("vert"): $10 \%$ of the error terms are now normal distributed with mean 20 and standard deviation 1.

3. (Bad) leverage points ("lev"): After the responses are generated using (8), $10 \%$ of the covariates are replaced with observations generated from independent $N(50,1)$ distributions.

To compare the performances of the different methods, we generate 100 independent samples of size $n=200$ from the three simulation designs and calculate the evaluation criteria given in Table 2 .

Table 2: Evaluation criteria

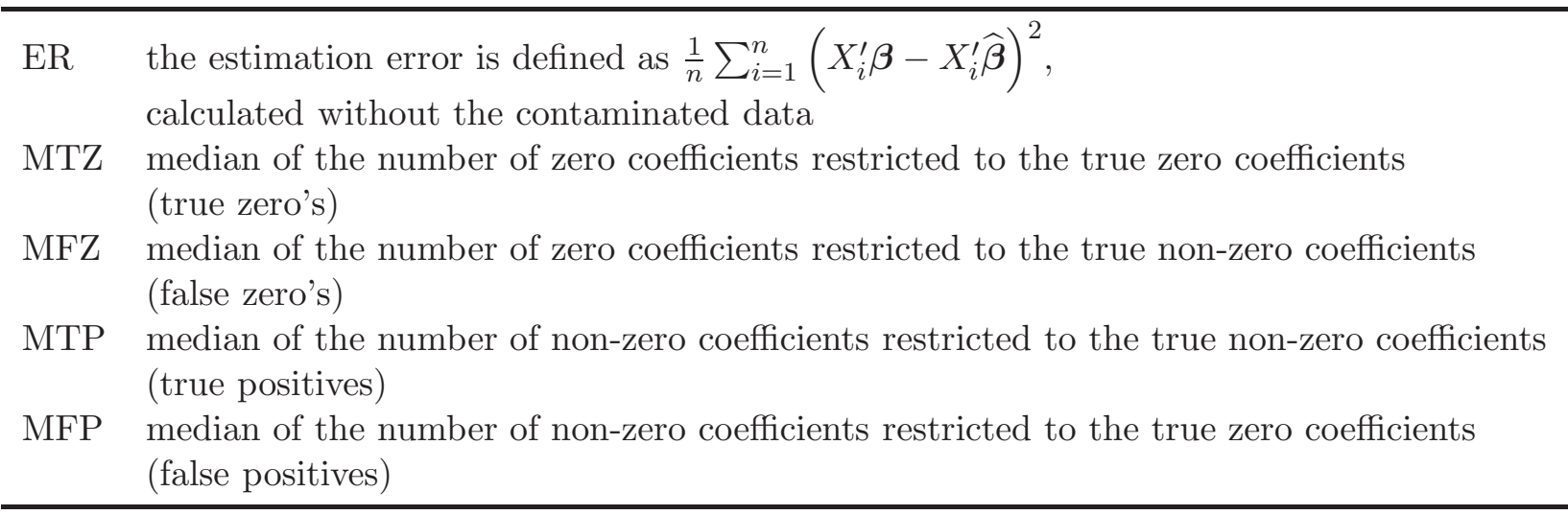

In Figure 1 the five evaluation criteria for the original nonnegative garrote are plotted. We also report on the performance of four criteria (the L-curve criterion, the BIC- and AIC-criteria and five fold cross-validation) to select the regularization parameter $\lambda$. The means and plus/minus 


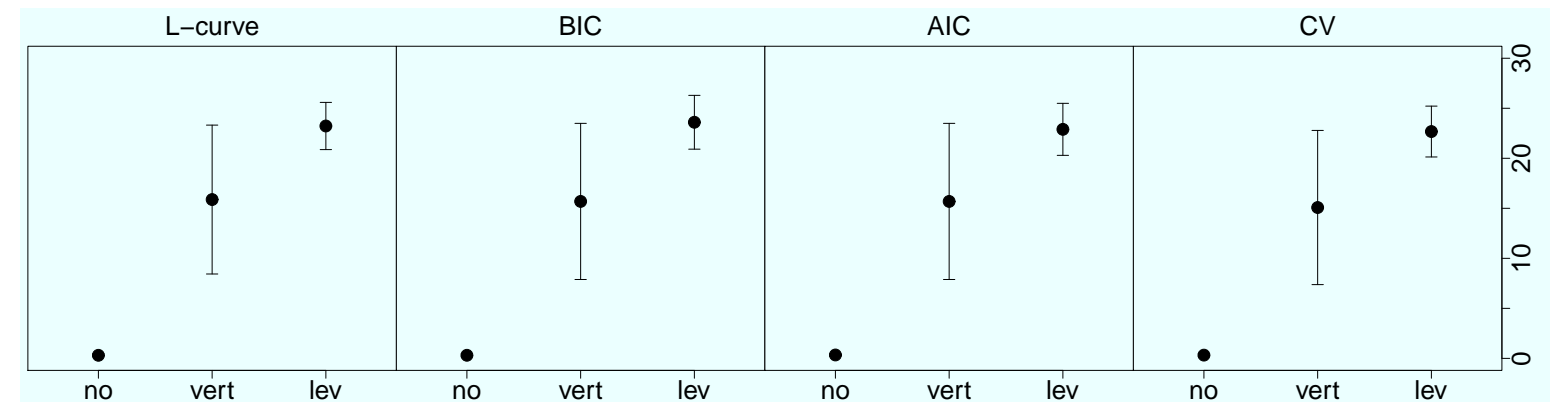

(a)

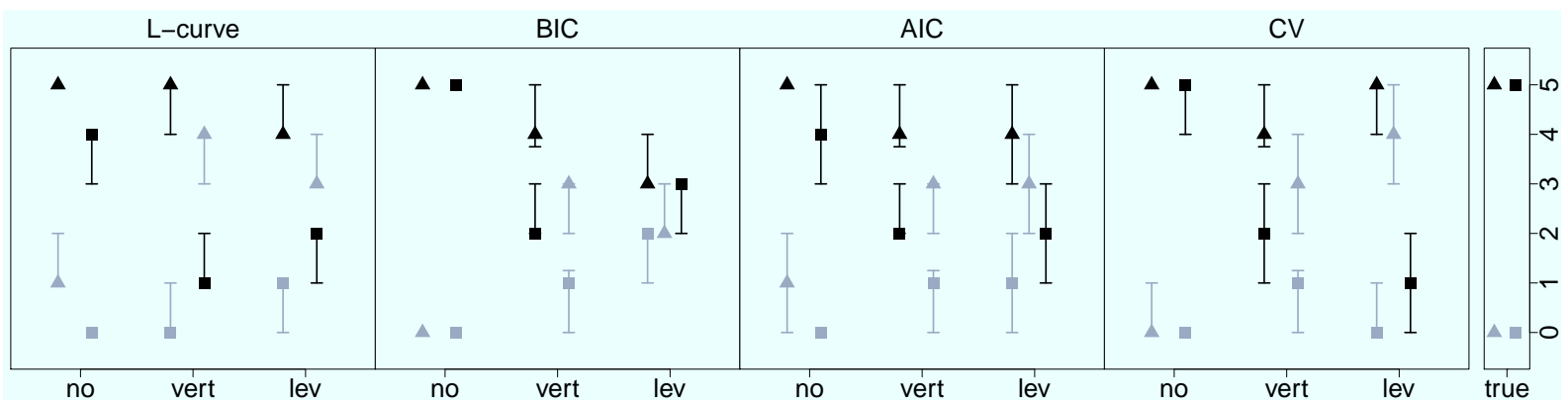

(b)

Figure 1: (a) Mean and standard deviation of the estimation error; (b) median, first and third

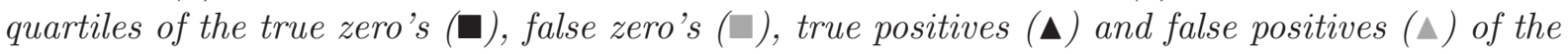
nonnegative garrote for no contamination ("no"), vertical outliers ("vert") and leverage points ("lev"). The optimal values are plotted in the side bar.

the standard deviations of the estimation errors for each of the three contamination schemes are presented in Figure 1(a). This figure shows that the estimation error becomes very large if the data set is contaminated. Due to the large scale of the plots one can hardly notice any differences in performance of the four selection criteria, but no method really strongly dominates with respect to finite-sample performance. In Figure 1(b) the medians, first and third quartiles of the true positives, true zero's, false positives and false zero's are plotted. The nonnegative garrote works well if the MTP and MTZ are close to five and the MFP and MFZ are close to zero. If there is no contamination, this is satisfied, but if the data set is contaminated, the MTZ are too low and the MFP are too high. Furthermore, the nonnegative garrote sometimes puts the wrong coefficients equal to zero, since the MFZ is different from zero if the data set contains vertical outliers or leverage points.

In Figure 2 the MTP, MTZ, MFP and MFZ are mapped together with their first and third quartiles for the M-, LTS- and S-nonnegative garrote. This is done for the three different contamination schemes and when the OLS-, S-, LTS- and $\tau$-estimators are used for the initial estimator. Moreover, four data-driven criteria are used to select the regularization parameter $\lambda$, namely the robust L-curve criterion, RCV, RAIC and RBIC. The robust variable selection methods are optimal when the MTP and MTZ are close to five and the MFP and MFZ are close to zero.

Figure 2 shows that if RCV or RAIC are used to select the regularization parameter, the MFPs are too high and the MTZs are too low. Thus, we conclude that these criteria select models with too many variables. Furthermore, it can be seen that if the data set is contaminated and the OLS-estimator is used for the initial estimator, all the robust nonnegative garrote methods put the wrong coefficients equal to zero, since the MFZs are different from zero. The MFPs are also 


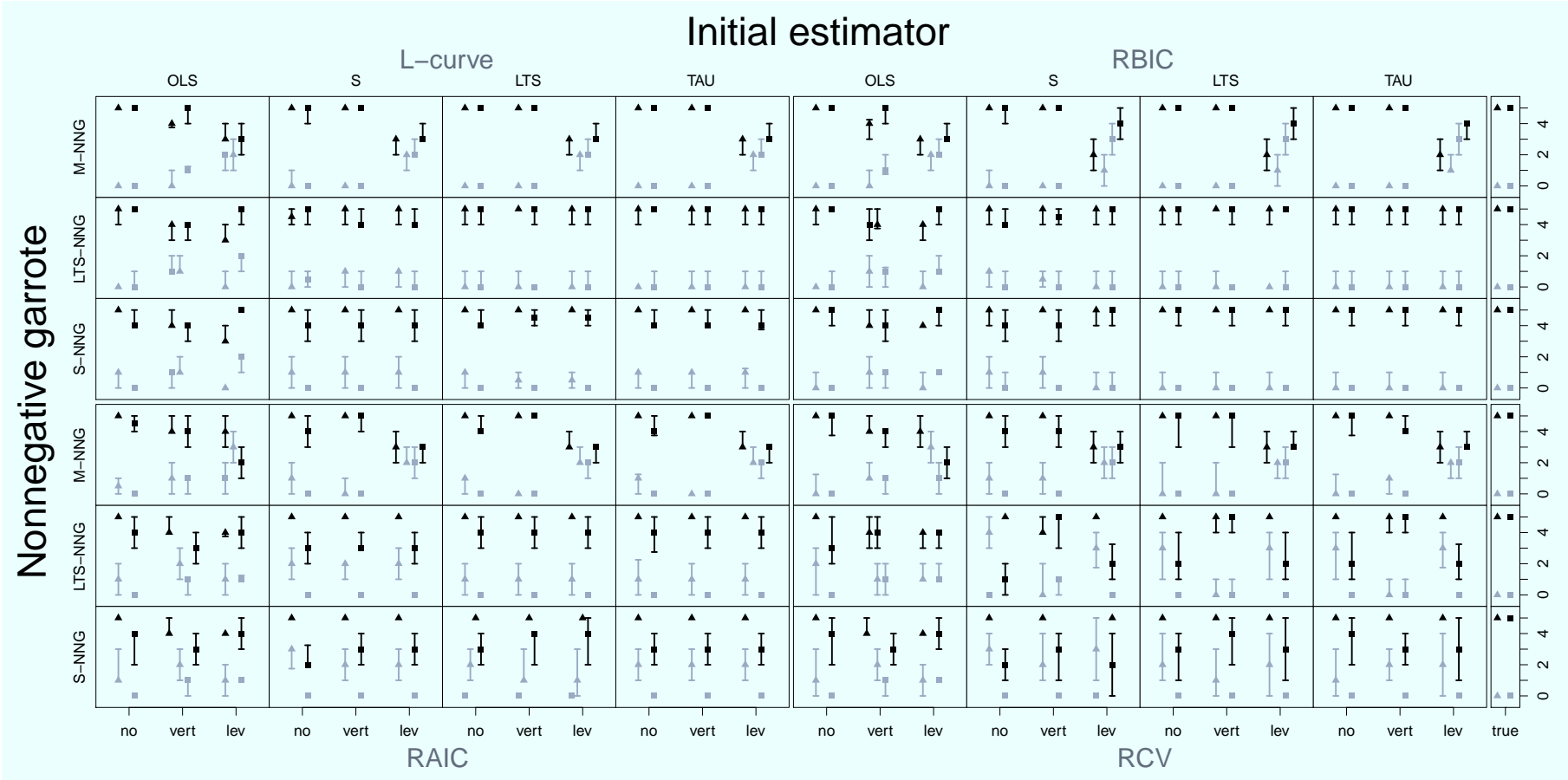

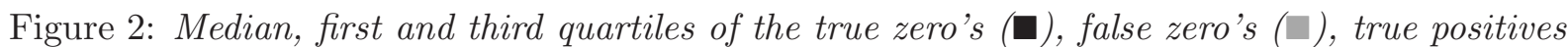
$(\mathbf{\Delta})$ and false positives ( $\Delta$ ) for no contamination ("no"), vertical outliers ("vert") and leverage points ("lev") and the robust alternatives of the nonnegative garrote. The optimal values are plotted in the side bar.

too high, while the MTZs and MTPs are too low. It is therefore necessary to use a robust initial estimator. If the S-estimator is used as initial estimator, the performances improve, but the MFPs and MTZs remain too high and too low respectively. Since the algorithms of the LTSand $\tau$-estimator return in $\mathrm{R}$ estimates with high efficiency, these methods perform better as initial estimator than the S-estimator. In addition, the performances of the different estimation methods for the shrinkage factors show that the M-nonnegative garrote performs better than the S- and LTS-nonnegative garrote when the data set is uncontaminated or contains vertical outliers. But if the data set contains leverage points, the MFZs and MFPs for the M-nonnegative garrote are too high and the MTZs and MTPs are too low. The LTS- and S-nonnegative garrote perform well as estimation methods for the shrinkage factors.

We conclude that it is better to use a robust and highly efficient initial estimator, such as the LTS- and $\tau$-estimator. In addition, the LTS- and S-nonnegative garrote are robust to vertical outliers and leverage points and have, for this simulation study, the best results for the MTP, MFP, MTZ and MFZ, when the L-curve criterion or RBIC are used to select the regularization parameter. We next look at the estimation errors of these methods.

In Figure 3 the means and standard deviations of the estimation errors are plotted for the M-, LTS- and S-nonnegative garrote and for the three contamination schemes. This figure shows that the M-nonnegative garrote has the lowest mean estimation errors, when the data set is uncontaminated or contains vertical outliers. When there are leverage points in the data set, this method breaks down. It can also be seen that the mean estimation errors of the Snonnegative garrote are smaller than these of the LTS-nonnegative garrote. Furthermore, there is little difference between the mean estimation errors of the S-nonnegative garrote, when the L-curve criterion or the RBIC are used to select the regularization parameter. Also the mean 


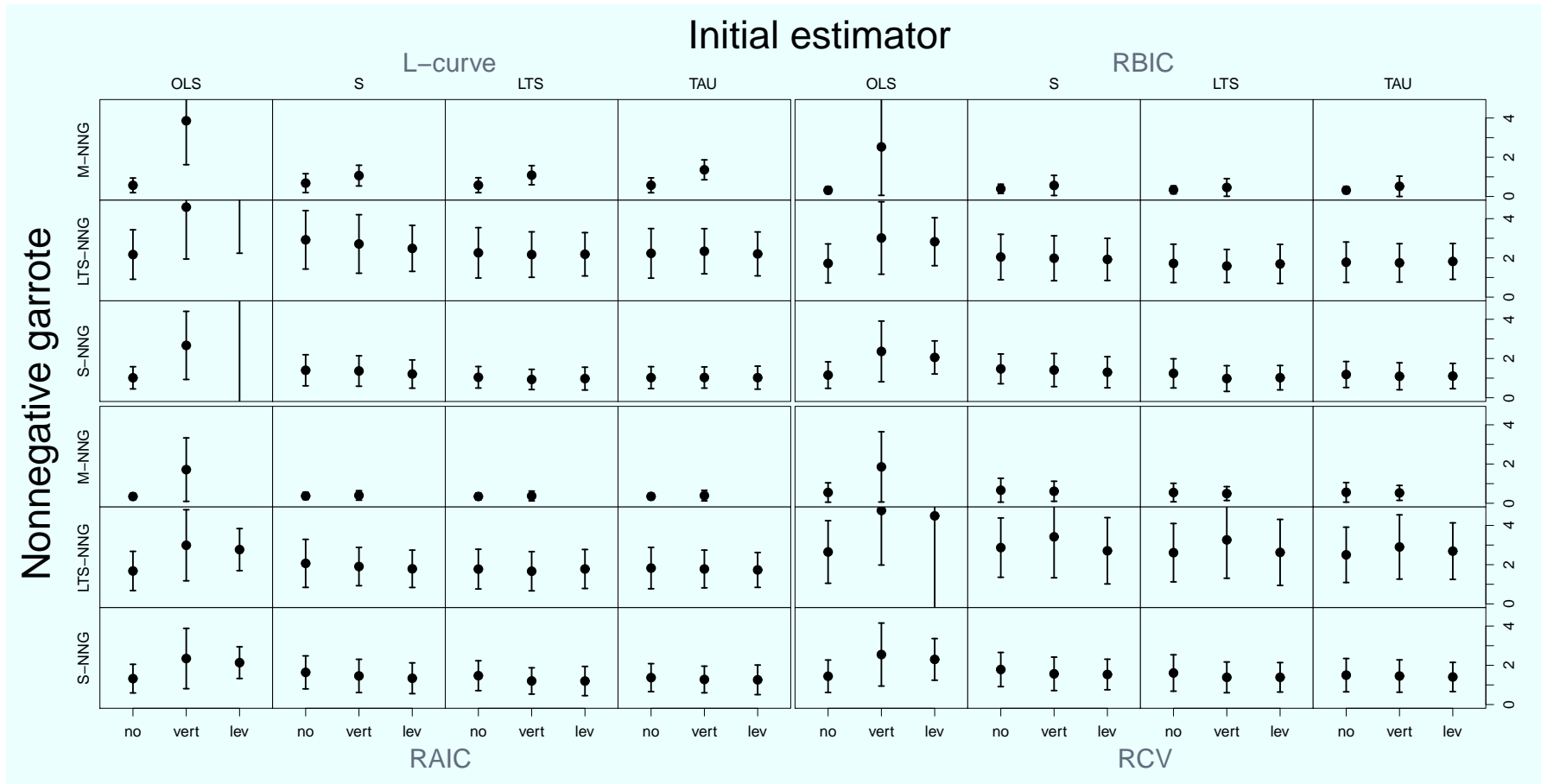

Figure 3: Mean and standard deviation of the estimation error for no contamination (" $n o$ "), vertical outliers ("vert") and leverage points ("lev") and the different robust nonnegative garrote methods.

estimation errors of the S-nonnegative garrote are comparable when the LTS- or the $\tau$-estimator are used as initial estimator.

Since the S-nonnegative garrote gives the best results for this simulation study, we want to improve this method to increase the efficiency. This is the aim of Section 4.

\section{The MM-nonnegative garrote}

The MM-nonnegative garrote combines the S- and M-nonnegative garrote, so that the MMnonnegative garrote shrinkage factors are robust to outliers and highly efficient when the error terms have a normal distribution. The MM-nonnegative garrote starts with computing the Snonnegative garrote shrinkage factors $\widehat{\mathbf{c}}^{0}$ and the M-scale of the residuals $\mathbf{r}_{S}\left(\widehat{\mathbf{c}}^{0}\right)$, using loss function $\rho_{0}$ and regularization parameter $\lambda_{0}$, and then uses these estimates as starting point for the M-nonnegative garrote with loss function $\rho_{1}$ and regularization parameter $\lambda_{1}$. The initial shrinkage factors $\widehat{\mathbf{c}}^{0}$ are thus obtained by solving

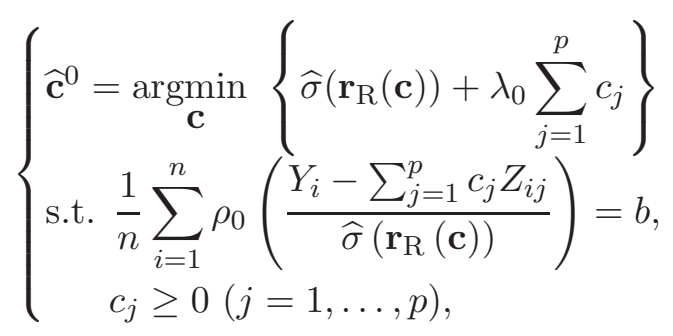


and the MM-nonnegative garrote shrinkage factors $\widehat{\mathbf{c}}$ are found by solving

$$
\left\{\begin{array}{l}
\widehat{\mathbf{c}}=\underset{\mathbf{c}}{\operatorname{argmin}}\left\{\sum_{i=1}^{n} \rho_{1}\left(\frac{Y_{i}-\sum_{j=1}^{p} c_{j} Z_{i j}}{\widehat{\sigma}\left(\mathbf{r}_{\mathrm{R}}\left(\widehat{\mathbf{c}}^{0}\right)\right)}\right)+n \lambda_{1} \sum_{j=1}^{p} c_{j}\right\} \\
\text { s.t. } c_{j} \geq 0(j=1, \ldots, p) .
\end{array}\right.
$$

The algorithms of the S- and M-nonnegative garrote can thus be used to compute the MMnonnegative garrote shrinkage factors. Possible loss functions for $\rho_{0}$ and $\rho_{1}$ are loss functions of the Tukey's bi-square family with $d$ equal to $1.547(b=0.5)$ and 5.182 respectively.

\section{Simulation study: part II}

In this section we compare the MM-nonnegative garrote method with other available methods. We consider three simulation settings and report on the performances of the following methods:

- NNG: the original (nonrobust) nonnegative garrote method;

- MM-NNG: the MM-nonnegative garrote method described in Section 4;

- Sparse LTS: the Sparse least trimmed squares method of Alfons et al. (2013);

- B-RLARS: the Bootstrapped RLARS method of Khan et al. (2007);

- WLAD-LASSO: the Weighted LAD-LASSO method of Arslan (2012);

- ESL-LASSO: the Exponential Squared Loss LASSO method of Wang et al. (2013).

The first setting is the simulation example of Section 3. The second simulation example is a latent factor model with fifty covariates taken from Khan et al. (2007). The third simulation setting is a linear model with one strong component and nine weak or non-relevant components.

For each of the three simulation designs, five contamination schemes as considered in Khan et al. (2007), are applied:

1. No contamination.

2. $10 \%$ of the error terms come from a slash distribution: $N(0,1) /$ Uniform $(0,1)$. (This distribution has heavier tails than a normal distribution and its mean and variance do not exist.)

3. Same as in $\mathbf{2}$, but the $10 \%$ contaminated observations have high-leverage points generated from independent $N(50,1)$ distributions.

4. $10 \%$ of the error terms come from a normal distribution: $N(20,1)$.

5. Same as in 4, but the $10 \%$ contaminated observations have high-leverage points generated from independent $N(50,1)$ distributions.

With these contamination schemes, leverage points and different types of vertical outliers are induced in the samples.

To investigate the performances of the robust methods, we generate for each simulation setting 100 independent samples from the five contamination schemes. The evaluation criteria of Table 2 are used to summarize the results. For the MM-NNG, we use the $\tau$-estimator as initial estimator and, as proposed in the literature, the MM-estimator is used as initial estimator for the ESL-LASSO method. MM-regression is also used to estimate the selected covariates for the B-RLARS. In addition, values for the regularization parameters are obtained by minimizing the 
robust BIC-criterion. It should be mentioned that for the ESL-LASSO method we first tried the choice $\lambda_{n j}=n^{-1} \log (n)\left|\widetilde{\beta}_{n j}\right|^{-1}$ where $\widetilde{\beta}_{n j}$ is the initial robust estimator for $\beta_{j}$, as recommended in Wang et al. (2013). However this led to unsatisfactory results, with an apparently too large value of the regularization parameter(s). In our simulation study we therefore in addition replace the fixed factor $n^{-1} \log (n)$ by a global regularization parameter $\lambda$ which we choose via the robust BIC criterion. Further, in the first and third example, $50 \%$ of the observations are used to estimate the Sparse LTS, while in the second example $75 \%$ of the observations are used, so that we have the same breakdown point for each method.

In a supplemental material part, we summarize simulation results when the contamination level is increased from $10 \%$ to $20 \%$.

\subsection{Example 1}

We take again the linear model of Section 3, but use other contamination schemes for this comparison of performances of the different methods.

In Figure 4 the medians, first and third quartiles of the true positives, true zero's, false positives and false zero's are plotted for the five contamination schemes. Recall that the variable selection methods work well when the MTP and MTZ are close to five and the MFP and MFZ are close to zero. It can be seen that the MM-NNG performs equally well as the ESL-LASSO method in variable selection, and performs better than the other methods, because for all five contamination schemes the MFPs are equal to zero and the MTZs are equal to five. The Sparse LTS selects the correct variables, but the MFPs are rather big and the MTZs are small. Further, it seems that the B-RLARS and WLAD-LASSO have the same problem as the Sparse LTS when the data contain leverage points (contamination schemes 3 and 5). In Figure 5 the means and standard deviations of the estimation errors are plotted. This figure shows that the estimation errors of the MM-NNG garrote are comparable with these of the NNG, when the data are not contaminated. When the data contain outliers, the estimation errors of the NNG are very large (and outside the boundary of the plotting range). This is indicated by the vertical lines in its plot. Furthermore, the mean of the estimation errors of the MM-NNG are lower than or comparable with these of the other methods.

The boxplots of the differences between the estimated values and the true values of the coefficients for the first three contamination schemes are presented in Figures 6, 7 and 8. Recall that only variables 1, 2, 5, 6 and 10 are included in the true model. From these figures, it can be seen that the MM-NNG performs very well and that the NNG performs very bad, when the data are contaminated. The MM-NNG and the ESL-LASSO methods have a comparable performance, with a slightly higher variability for the ESL-LASSO method, which was also already noticeable from Figure 5. The B-RLARS and WLAD-LASSO select too many non-relevant variables when the data contain leverage points and the B-RLARS then also underestimates the tenth variable. In addition, the estimated values of the coefficients of the Sparse LTS are close to the true values, but it also selects too many non-relevant variables.

\subsection{Example 2}

This linear model with 6 latent variables is taken from Khan et al. (2007). We consider

$$
Y_{i}=L_{i 1}+L_{i 2}+\cdots+L_{i 6}+\sigma \epsilon_{i}
$$

where $L_{i 1}, \ldots, L_{i 6}$ and the error term $\epsilon_{i}$ are independent standard normal variables. The value $\sigma=\sqrt{6} / 3$, so that the signal-to-noise ratio is 9 . A set of $p=50$ covariates is created using 


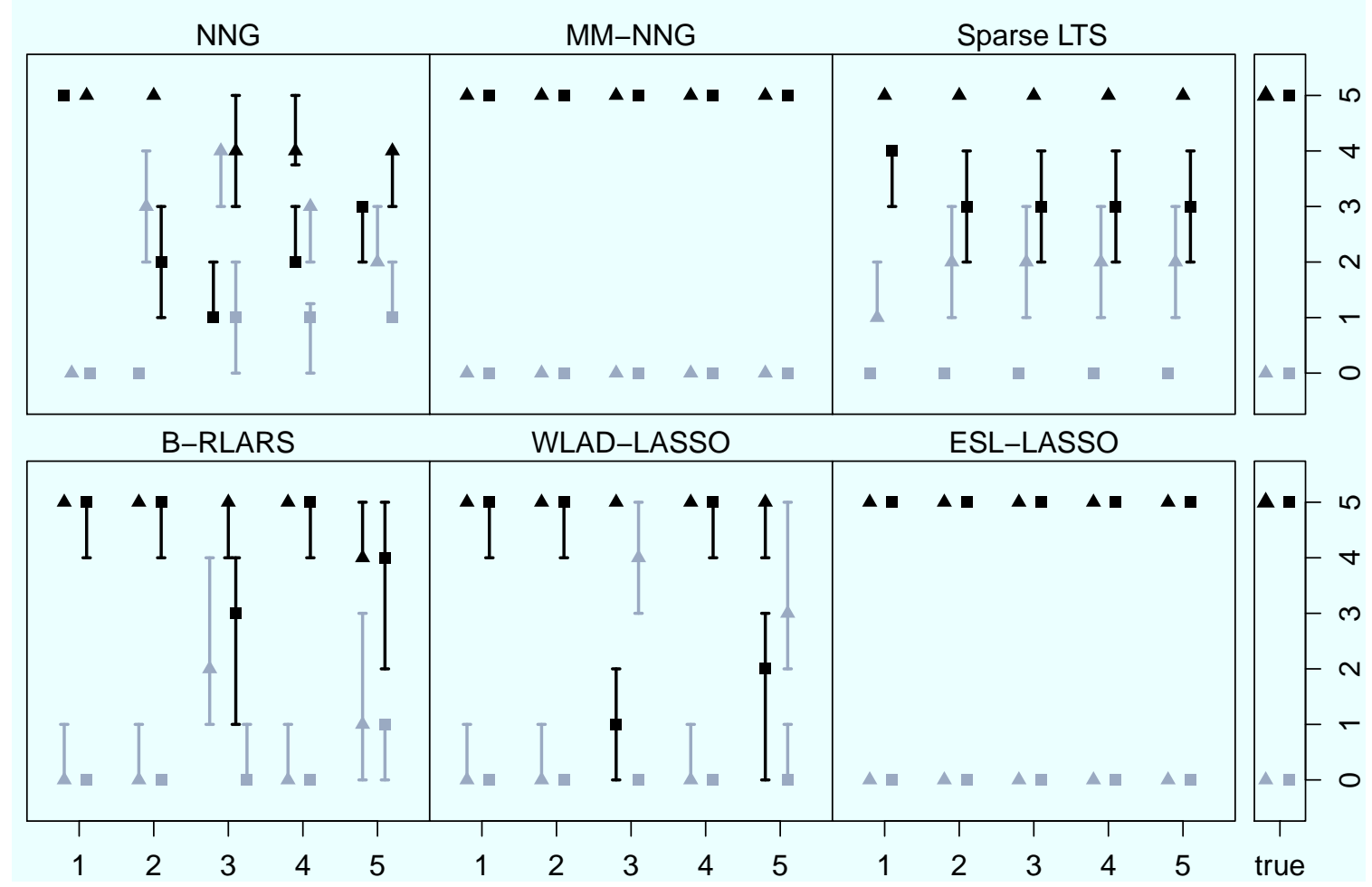

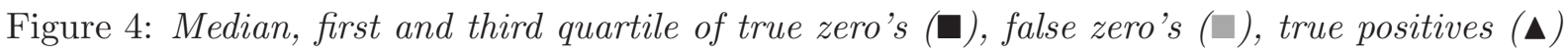
and false positives ( $\triangle$ ) for simulation example 1. The optimal values are $M T Z=5, M F Z=0$, $M T P=5$ and $M F P=0$ and they are plotted in the side bar.

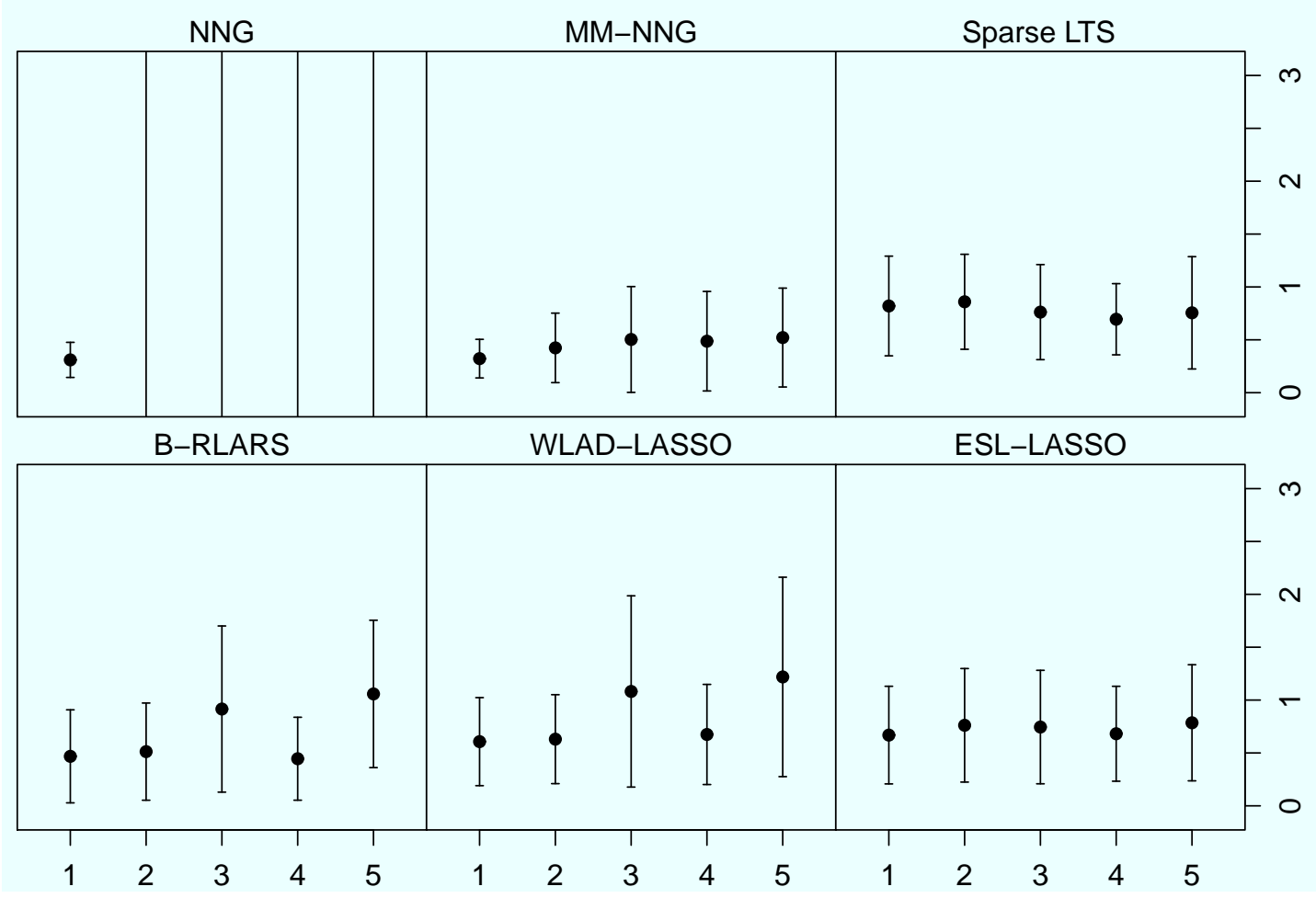

Figure 5: Mean and standard deviation of the estimation error for simulation example 1. 


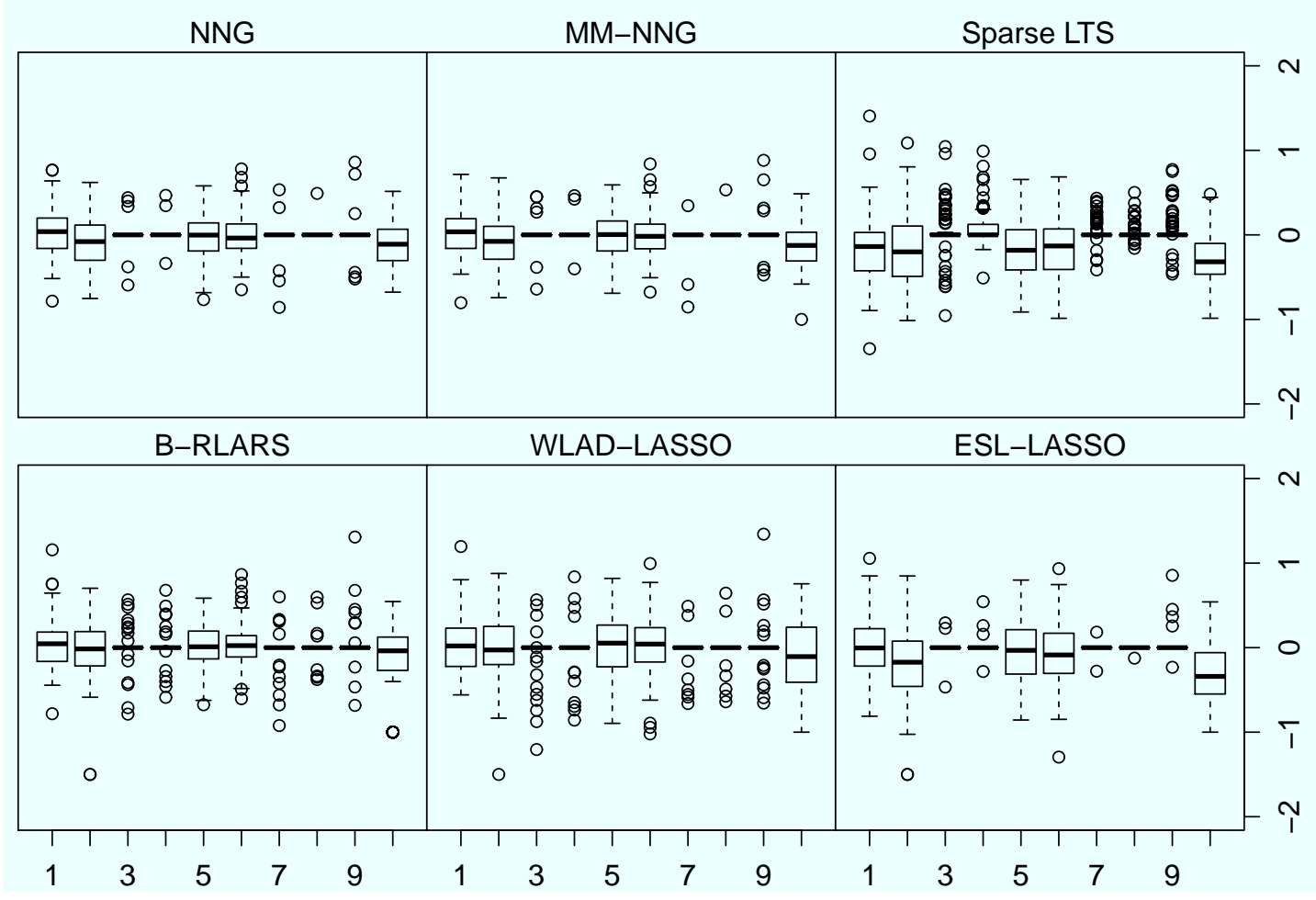

Figure 6: Difference between the estimated value and the true value of the coefficients of simulation example 1 and contamination scheme 1.

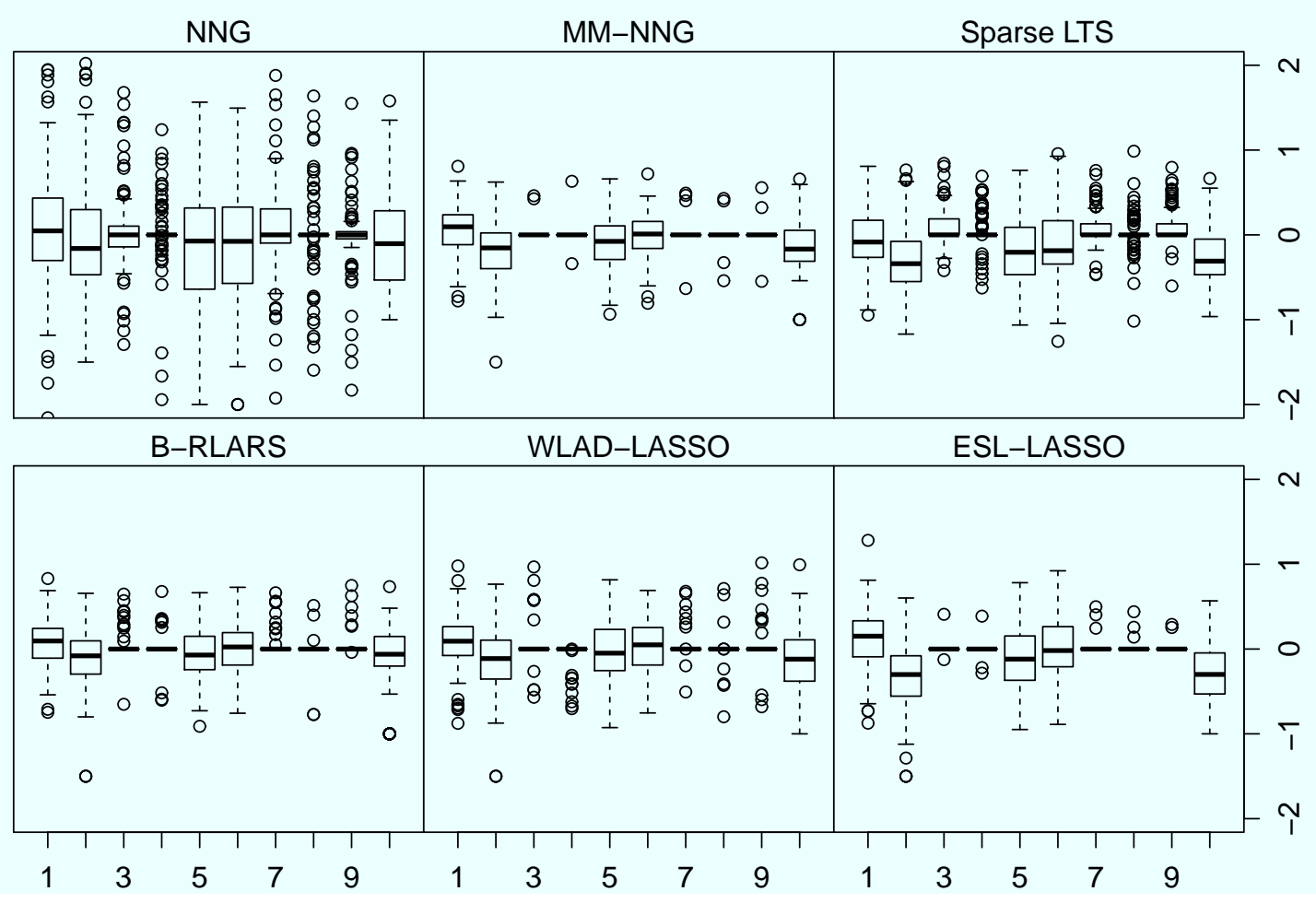

Figure 7: Difference between the estimated value and the true value of the coefficients of simulation example 1 and contamination scheme 2. 


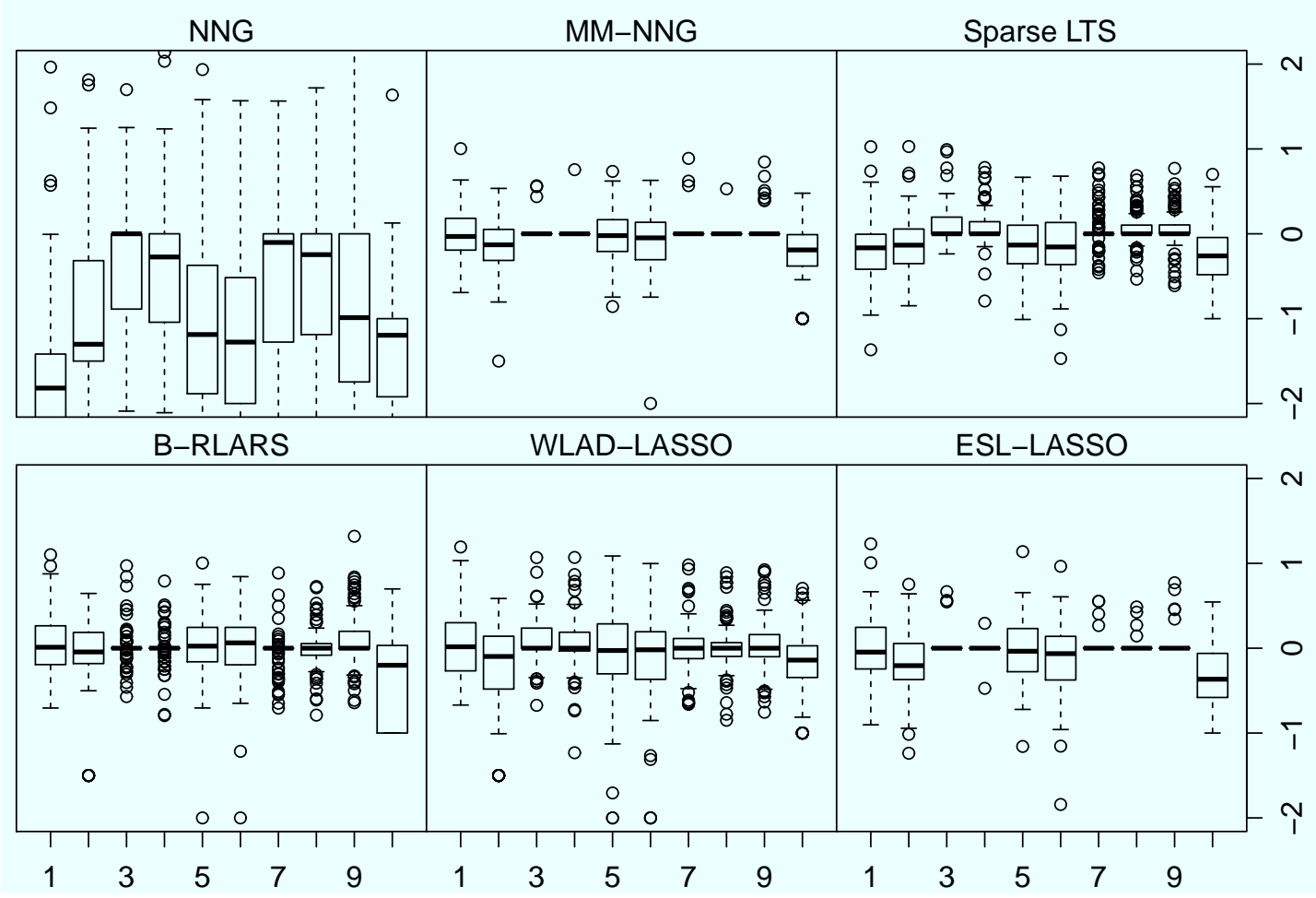

Figure 8: Difference between the estimated value and the true value of the coefficients of simulation example 1 and contamination scheme 3.

independent standard normal variables $E_{i 1}, \ldots, E_{i p}$,

$$
\begin{aligned}
X_{i j}= & L_{i j}+\tau E_{i j}, \quad j=1, \ldots, 6, \\
X_{i 7}= & L_{i 1}+\delta E_{i 7} \\
X_{i 8}= & L_{i 1}+\delta E_{i 8} \\
X_{i 9}= & L_{i 2}+\delta E_{i 9} \\
X_{i 10}= & L_{i 2}+\delta E_{i 10} \\
& \vdots \\
X_{i 17}= & L_{i 6}+\delta E_{i 17} \\
X_{i 18}= & L_{i 6}+\delta E_{i 18} \\
X_{i j}= & E_{i j}, \quad j=19, \ldots, 50,
\end{aligned}
$$

where $\tau=0.3$ and $\delta=5$ so that $\operatorname{Corr}\left(X_{i 1}, X_{i 7}\right)=\operatorname{Corr}\left(X_{i 1}, X_{i 8}\right)=\operatorname{Corr}\left(X_{i 2}, X_{i 9}\right)=\cdots=$ $\operatorname{Corr}\left(X_{i 6}, X_{i 18}\right) \approx 0.1878$. The covariates $X_{1}, \ldots, X_{6}$ are low noise perturbations of the latent variables, our target covariates, $X_{7}, \ldots, X_{18}$ are noise covariates correlated with the target covariates and $X_{19}, \ldots, X_{50}$ are independent noise covariates. The sample size $n$ is 150 .

In Figure 9 the medians, first and third quartiles of the true positives, false positives, true zero's and false zero's are plotted for the five simulation designs. The variable selection methods are optimal when the MTP is close to 6, the MTZ is close to 44 and the MFP and MFZ are close to 0 (see the vertical bar to the right indicating these values). We can draw the same conclusions as for the first simulation example, namely the MM-NNG method (and to a slightly lesser extent the ESL-LASSO method) performs better variable selection than the other methods. The Sparse LTS selects the correct variables, but again the MFPs are rather big and the MTZs are small. 


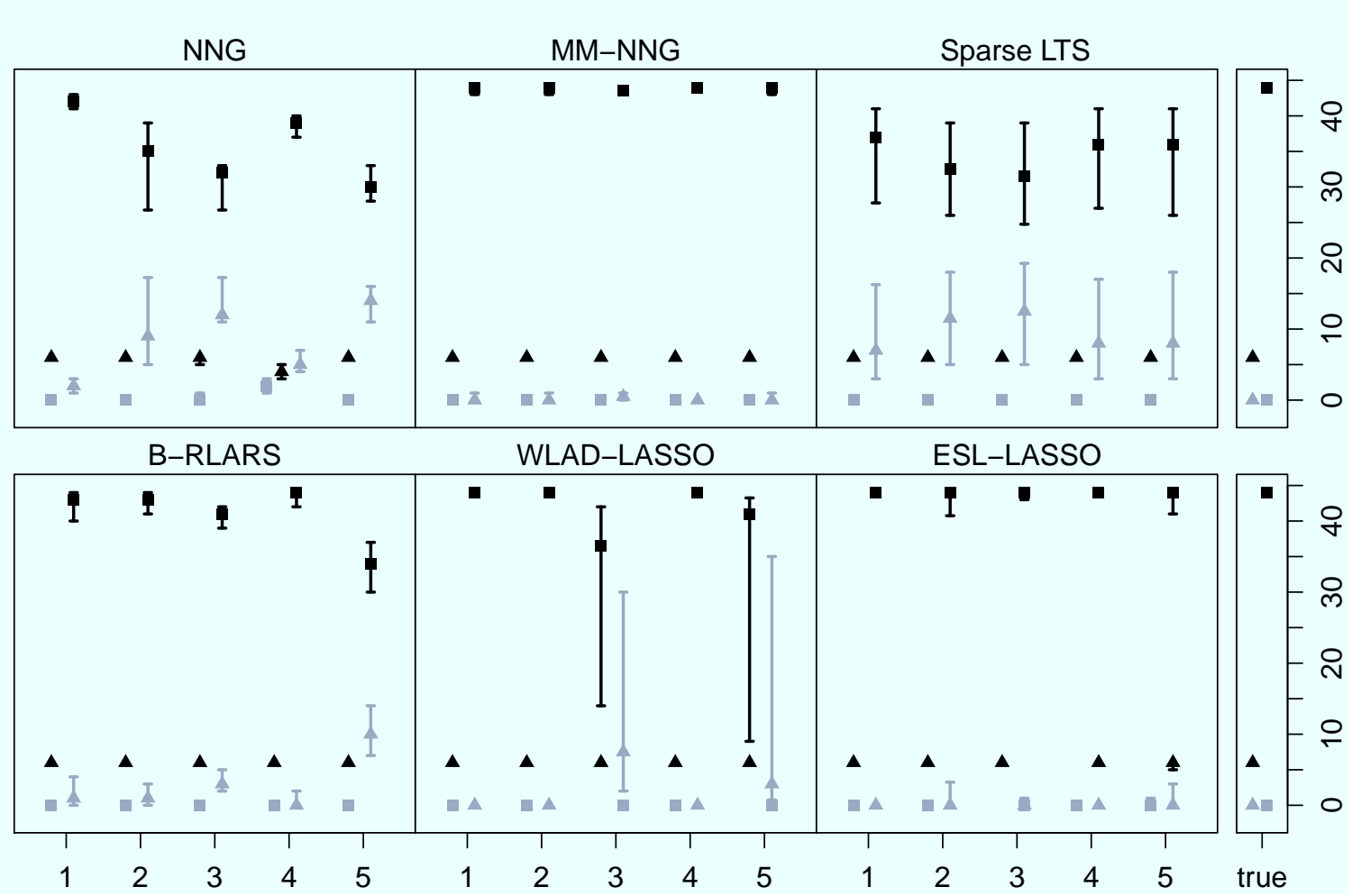

Figure 9: Median, first and third quartile of the true zero's (ם), false zero's ( $\square$ ), true positives $(\Delta)$ and false positives $(\Delta)$ for simulation example 2. The optimal values are $M T Z=44, M F Z=$ $0, M T P=6$ and $M F P=0$ and they are plotted in the side bar.

The same problems occur for the B-RLARS and WLAD-LASSO: they select too many variables when the data contain leverage points (contamination schemes 3 and 5 ).

The means and standard deviations of the estimation errors of the different methods are shown in Figure 10. The estimation errors of the MM-NNG, Sparse LTS, B-RLARS and WLADLASSO are comparable, with B-RLARS having the smallest errors. The estimation errors of the MM-NNG, B-RLARS, WLAD-LASSO and ESL-LASSO are bigger when the data contain vertical outliers and leverage points (simulation designs 3 and 5), while the estimation errors of the Sparse LTS are similar (but overall a bit higher) for all the contamination schemes.

\subsection{Example 3}

In this simulation model we consider a linear model with 10 covariates, where one covariate is a strong component and the others are weak or non-relevant components. The regression model is given by

$$
Y_{i}=X_{i}^{\prime} \boldsymbol{\beta}+\sigma \epsilon_{i}=5 X_{i 1}+0.5 X_{i 2}+0.5 X_{i 3}+0.5 X_{i 4}+\sigma \epsilon_{i},
$$

where the covariates $X_{i 1}, \ldots, X_{i 10}$ are generated from a multivariate normal distribution with mean 0 and $\operatorname{Corr}\left(X_{i j}, X_{i k}\right)=0.5^{|j-k|}$ for $j, k=1, \ldots, 10$. The error term $\epsilon_{i}$ is standard normal distributed and $\sigma=2.25$, so that the signal-to-noise ratio is equal to $\frac{164}{27} \approx 6.07$. We use a sample size of $n=200$.

The medians, first and third quartiles of the true positives, true zero's, false positives and false zero's are plotted for the five contamination schemes in Figure 11. The variable selection methods are optimal when the MTP is close to 4, the MTZ is close to 6 and the MFP and MFZ are close 


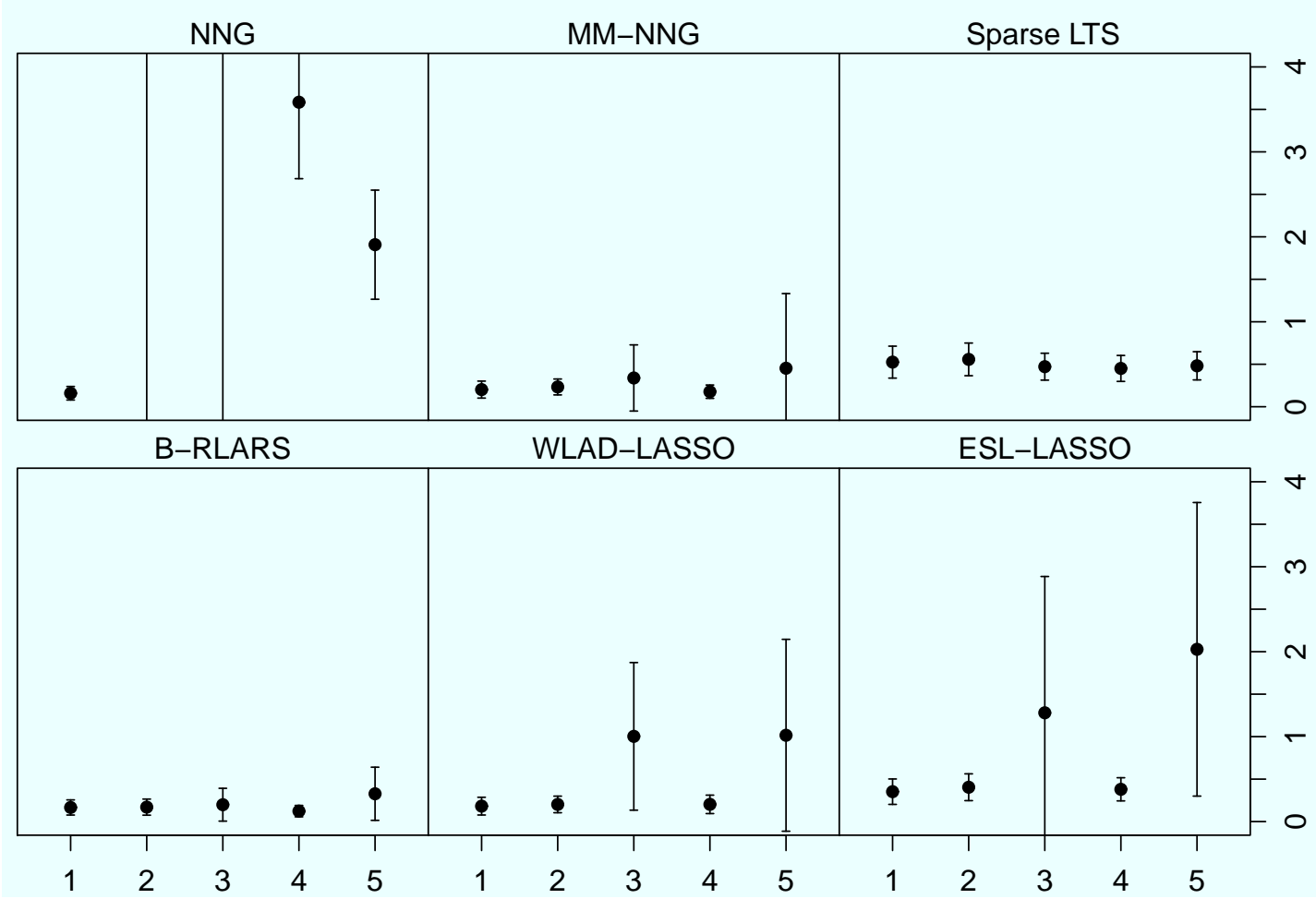

Figure 10: Mean and standard deviation of the estimation error for simulation example 2.

to 0. This figure shows that the MM-NNG, B-RLARS, WLAD-LASSO and ESL-LASSO all put too many coefficients equal to zero. When the data contain leverage points, the B-RLARS and WLAD-LASSO sometimes put the wrong coefficients equal to zero. The Sparse LTS selects the relevant covariates, but it also selects some non-relevant covariates.

Figure 12 shows the means and standard deviations of the estimation errors of the different methods. The estimation errors of all robust variable selection methods are comparable when there is no contamination or when the data contain vertical outliers. When the data also contain leverage points, the estimation errors of the B-RLARS are larger than these of the MM-NNG, Sparse LTS, WLAD-LASSO and ESL-LASSO.

In Figures 13, 14 and 15, the boxplots of the differences between the estimated values and the true values of the coefficients for contamination schemes 1,4 and 5 are presented. Recall that only covariates $1,2,3$ and 4 are relevant, with $X_{1}$ being the strongest component among these. From these figures it can be seen that the MM-NNG, B-RLARS, WLAD-LASSO and ESL-LASSO sometimes underestimate the coefficients of the weak covariates. The Sparse LTS does not have this problem, but it selects too many non-relevant components.

\subsection{Computation times}

The simulations are performed in R. For the Sparse LTS, we used the function sparseLTS from the R-package robustHD; for B-RLARS, the R-code available at $h t t p: / /$ users.ugent.be/ svaelst/software/RLARS.html is used and for ESL-LASSO, we used a matlab-code received from the authors. For the NNG, MM-NNG and WLAD-LASSO, we used our own code. Most of MM-NNG is implemented in C++ and the NNG and WLAD-LASSO are implemented in R. Computer codes for the MM-NNG method written by the authors are available on the site http://wis.kuleuven.be/stat/stat-inferen/codes. 


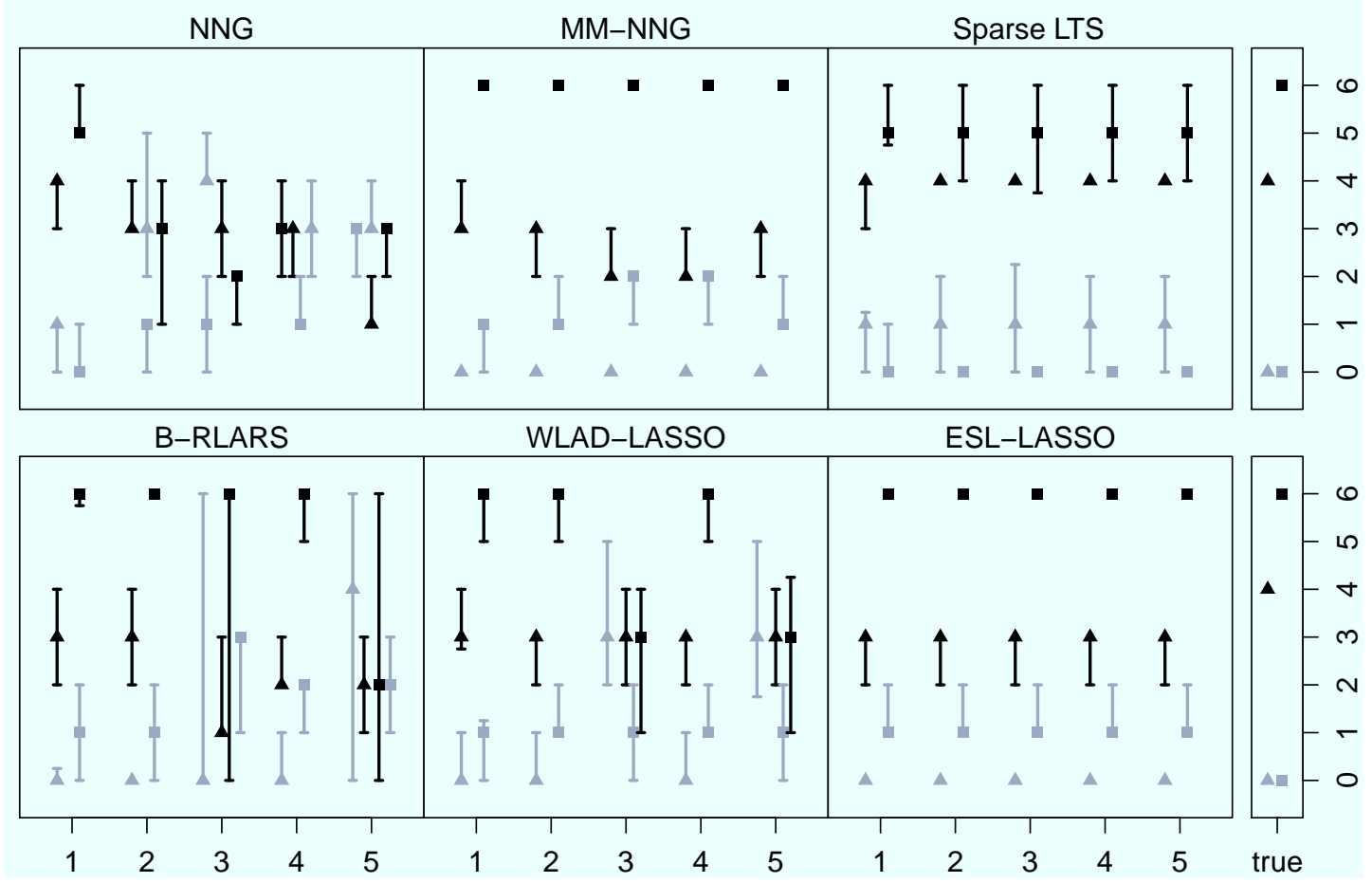

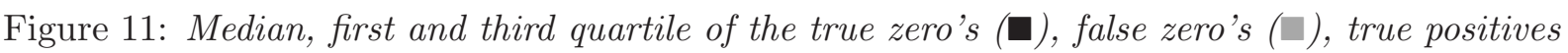
$(\mathbf{\Delta})$ and false positives ( $\triangle$ ) for simulation example 3. The optimal values are $M T Z=6, M F Z=$ $0, M T P=4$ and $M F P=0$ and they are plotted in the side bar.

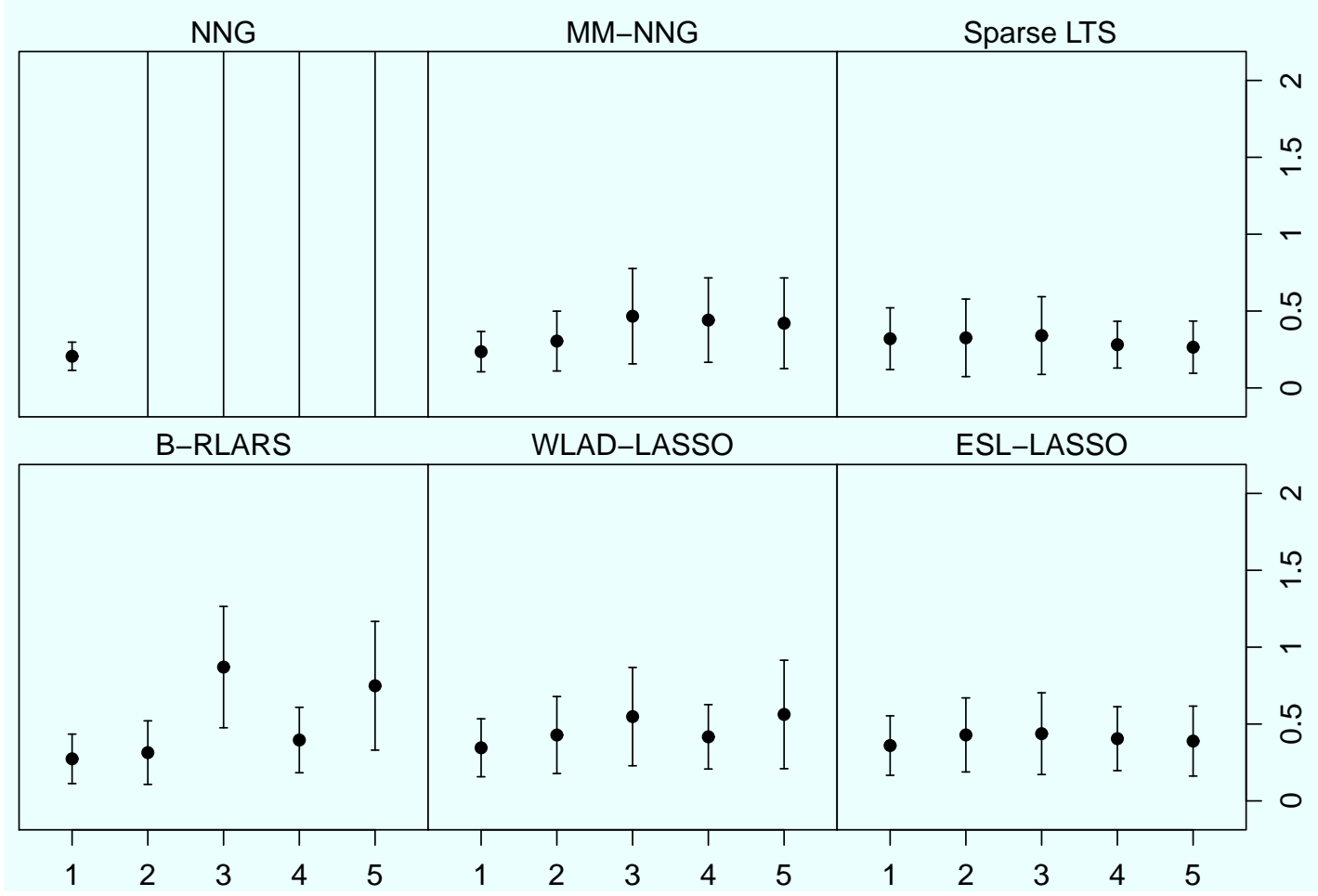

Figure 12: Mean and standard deviation of the estimation error for simulation example 3. 


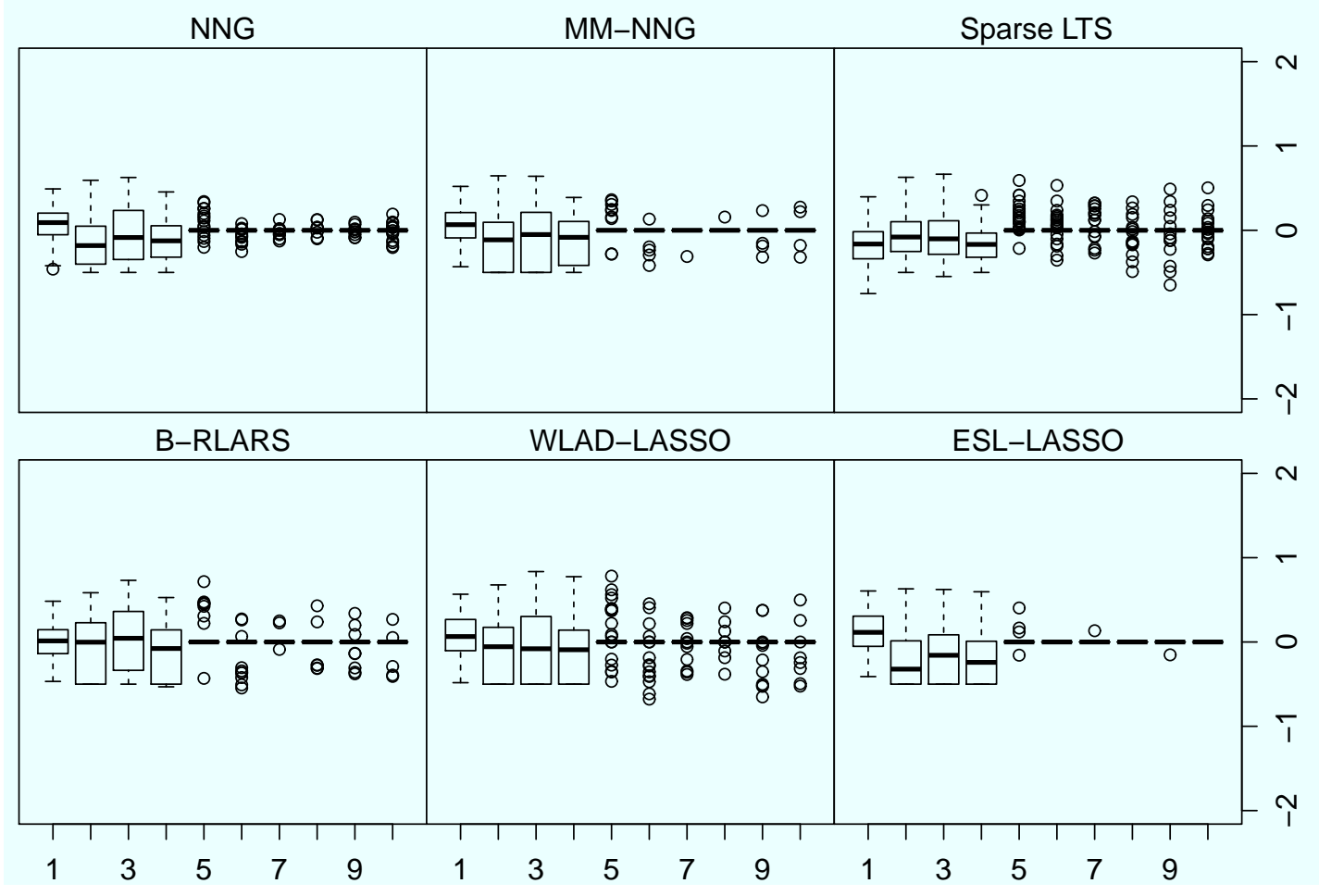

Figure 13: Difference between the estimated value and the true value of the coefficients of simulation example 3 and contamination scheme 1.

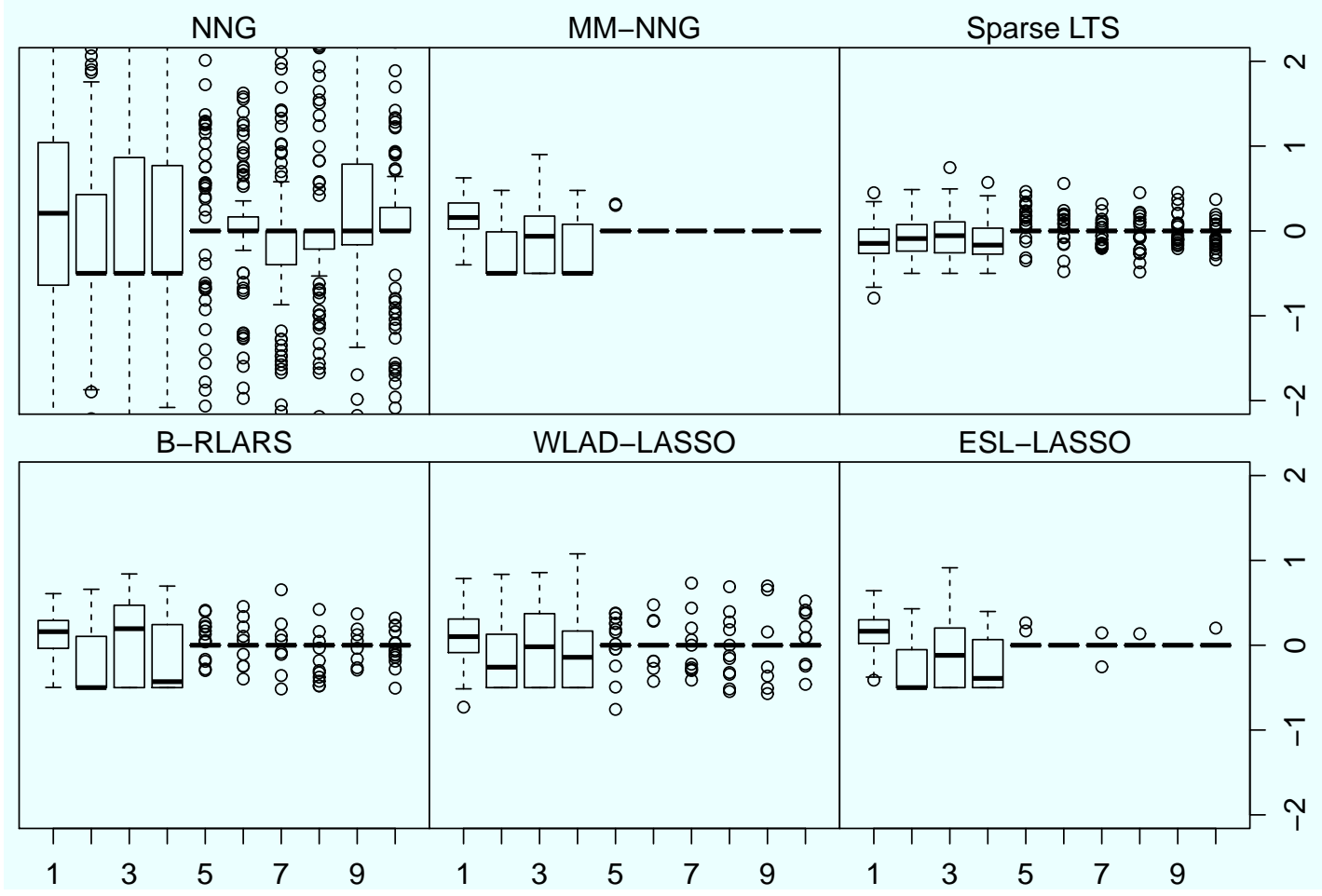

Figure 14: Difference between the estimated value and the true value of the coefficients of simulation example 3 and contamination scheme 4. 


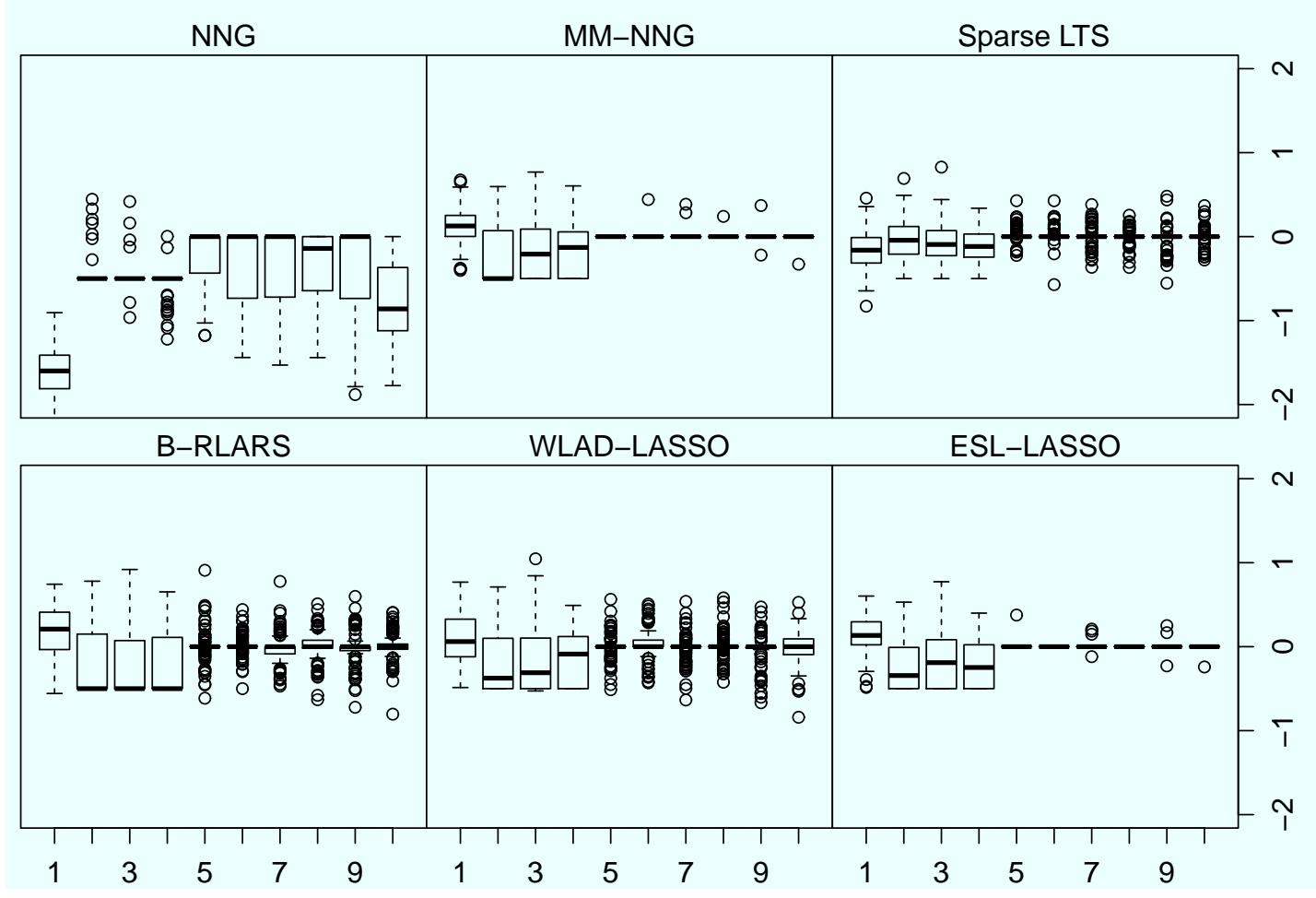

Figure 15: Difference between the estimated value and the true value of the coefficients of simulation example 3 and contamination scheme 5.

The computation times for the different methods are summarized in Table 3 . They are averaged over the five contamination schemes and over the 100 runs in each contamination scheme. The standard deviations are given in brackets. The computation times of the MM-NNG are higher than these of the other robust variable selection methods, but this is to be seen against the considerable gain in overall performance.

Table 3: Average computation times (in seconds) of the different variable selection methods for the three simulation examples.

\begin{tabular}{lcccccc}
\hline Example & NNG & MM-NNG & Sparse LTS & B-RLARS & WLAD-LASSO & ESL-LASSO \\
\hline 1 & 0.03 & 55.81 & 6.49 & 1.83 & 0.50 & 1.98 \\
& $(0.007)$ & $(6.51)$ & $(0.27)$ & $(0.07)$ & $(0.04)$ & $(0.22)$ \\
2 & 0.21 & 239.64 & 28.96 & 49.27 & 2.06 & 2.65 \\
& $(0.02)$ & $(20.62)$ & $(1.93)$ & $(3.93)$ & $(0.08)$ & $(0.13)$ \\
3 & 0.03 & 52.99 & 5.14 & 1.83 & 0.49 & 1.86 \\
& $(0.01)$ & $(5.66)$ & $(0.34)$ & $(0.03)$ & $(0.04)$ & $(0.16)$ \\
\hline
\end{tabular}

\section{Application to real data}

We consider the data set on air pollution and mortality in 60 metropolitan areas in the United States of Section 1 which is available at http://lib.stat.cmu.edu/DASL/Datafiles/SMSA.html. The response is age-adjusted mortality. The 14 covariates are mean January temperature (in degrees Farenheit), mean July temperature (in degrees Farenheit), relative humidity, annual 
rainfall (in inches), median education, population density, percentage of non whites, percentage of white collar workers, population, population per household, median income, hydrocarbon pollution potential, nitrous oxide pollution potential and sulfur dioxide pollution potential.

One observation had to be removed since it contains 2 missing values, and due to the skewness of the observations of the pollution variables, we consider the logarithm of them. In this section, the covariates and response variable are scaled to have median equal to zero and MAD equal to one, such that all variables are in a similar observation range. Since in this example the proportion of the sample size $n$ to the number of covariates $p$ is relatively small, we used as an initial estimator a further improvement of the MM-estimator. This was done for all methods that apply an MM-estimator.

Table 4 indicates which variables are selected by each of the methods. The selected variables are clearly different among the six methods.

The variables that are selected by all the robust variable selection methods are annual rainfall, median education, percentage of non whites and sulfur dioxide pollution potential. The Sparse LTS, WLAD-LASSO and ESL-LASSO select more variables than the MM-NNG and B-RLARS.

The main difference between the NNG and the robust variable selection methods is that the NNG selects nitrous oxide pollution potential instead of sulfur dioxide pollution potential.

Table 4: Mortality data: selected covariates.

\begin{tabular}{|c|c|c|c|c|c|c|c|}
\hline Variable & NNG & MM-NNG & Sparse LTS & B-RLARS & WLAD-LASSO & ESL-LASSO & $\begin{array}{c}\mathrm{NNG} \\
\text { (clean data) }\end{array}$ \\
\hline $\begin{array}{l}\text { JanTemp } \\
\text { JulyTemp } \\
\text { RelHum }\end{array}$ & $\checkmark$ & & $\sqrt{ }$ & & & $\checkmark$ & \\
\hline Rain & $\checkmark$ & $\checkmark$ & $\checkmark$ & $\sqrt{ }$ & $\checkmark$ & $\sqrt{ }$ & $\checkmark$ \\
\hline Education & & $\sqrt{ }$ & $\checkmark$ & $\sqrt{ }$ & $\checkmark$ & $\sqrt{ }$ & \\
\hline PopDensity & & $\checkmark$ & $\checkmark$ & & $\checkmark$ & $\checkmark$ & $\checkmark$ \\
\hline $\begin{array}{l}\text { \%NonWhite } \\
\% \text { WC }\end{array}$ & $\begin{array}{l}\checkmark \\
\checkmark\end{array}$ & $\checkmark$ & $\checkmark$ & $\sqrt{ }$ & $\checkmark$ & $\checkmark$ & $\begin{array}{l}\checkmark \\
\checkmark\end{array}$ \\
\hline $\begin{array}{l}\text { Pop } \\
\text { Pop/house }\end{array}$ & & & $\sqrt{ }$ & & & & \\
\hline Income & & & $\checkmark$ & & $\checkmark$ & & \\
\hline LogHCPot & & & & & $\checkmark$ & $\sqrt{ }$ & \\
\hline LogNoxPot & $\sqrt{ }$ & & & & & & \\
\hline LogSO2Pot & & $\sqrt{ }$ & $\sqrt{ }$ & $\sqrt{ }$ & $\sqrt{ }$ & $\sqrt{ }$ & $\sqrt{ }$ \\
\hline
\end{tabular}

Analyzing the data with a QQ-plot and a regression diagnostic plot which plots the classical Mahalanobis distance against a robust distance computed using the Minimum Covariance Determinant (MCD) estimator (see Figure 16), revealed five outliers. We removed these outliers from the data set and applied the NNG on the "clean" data set. The variables that are now selected by NNG can be found in the last column of Table 4. One can see that now the variable sulfur dioxide pollution potential is selected instead of nitrous oxide pollution potential. 


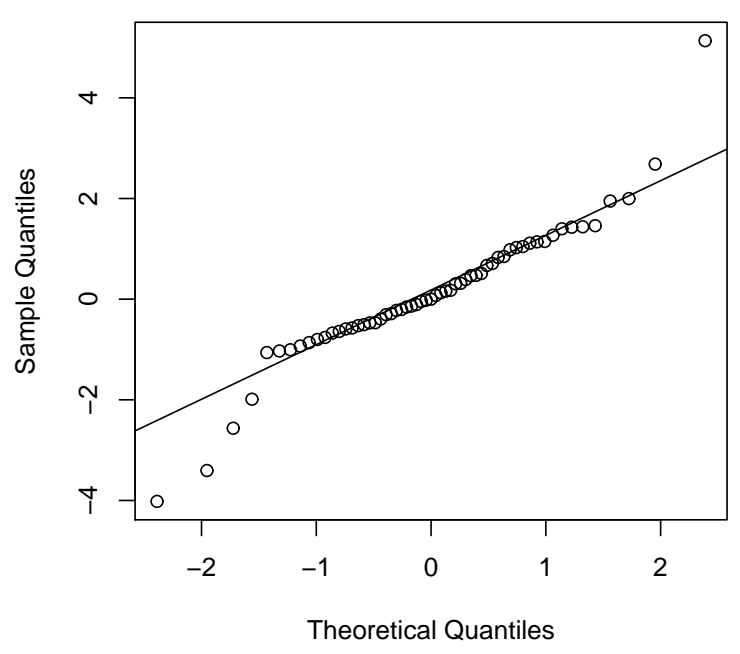

(a)

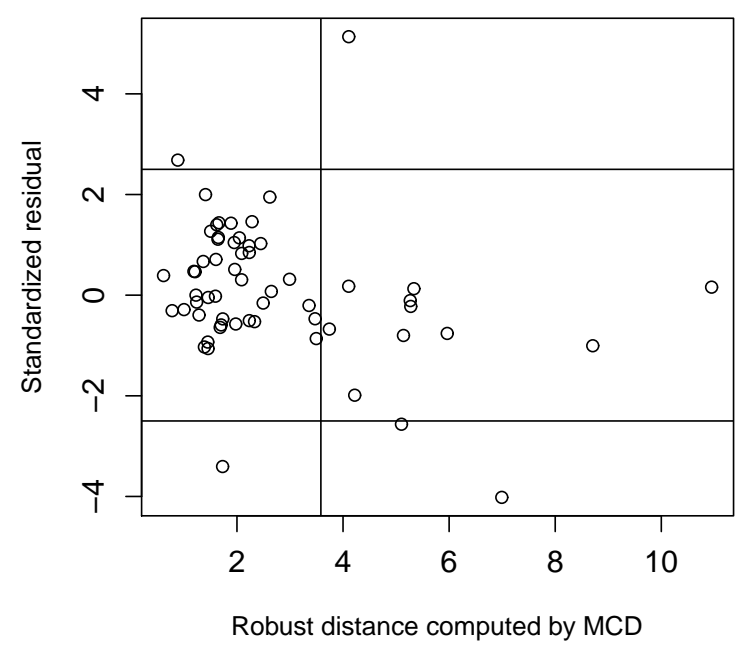

(b)

Figure 16: (a) QQ-plot and (b) Regression diagnostic plot for the data set on pollution and mortality.

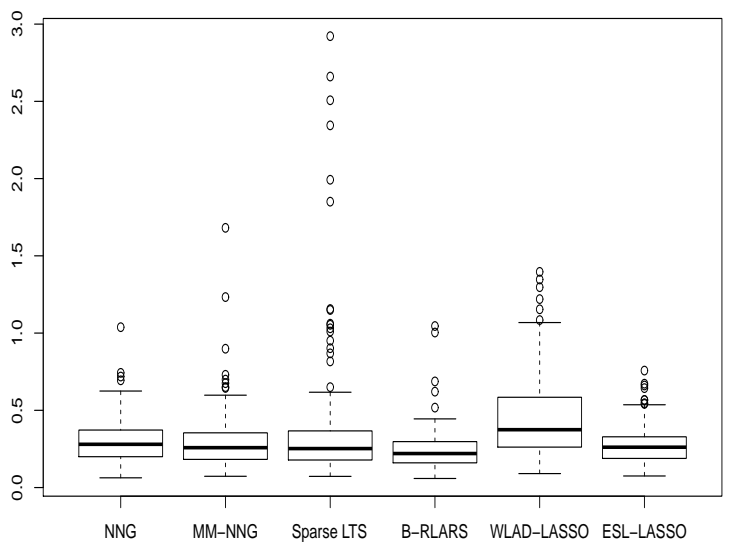

(a)

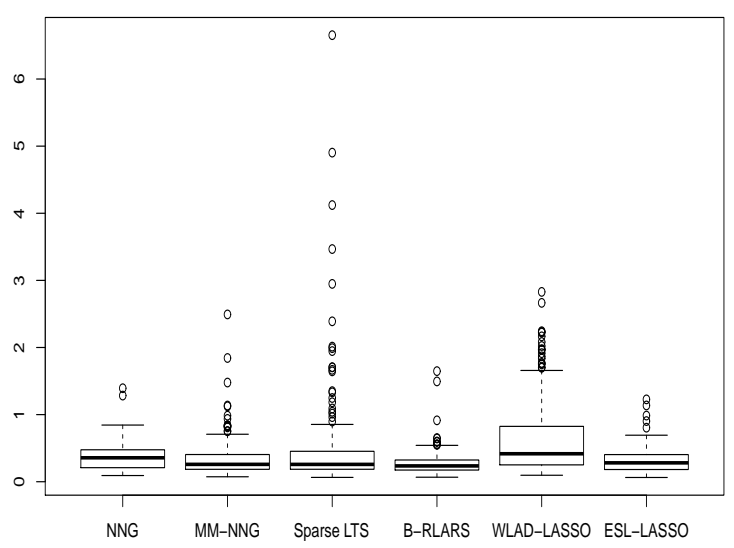

(b)

Figure 17: Boxplots of the means (left) and the standard deviations (right) of the prediction errors.

For further comparisons, we split randomly the data set into training and test sets of sizes 48 and 11 to obtain prediction errors for each variable selection method. For each observation $i$ in 
the test set that is not an outlier, the prediction error is given by

$$
\mathrm{PE}_{i}=\left(Y_{i}-\mathbf{X}_{i}^{\prime} \widehat{\boldsymbol{\beta}}^{*}\right)^{2}
$$

where $\widehat{\boldsymbol{\beta}}^{*}$ denotes the estimate of $\boldsymbol{\beta}$ based on the training set. Then, the mean and standard deviation of these prediction errors can be computed with

$$
\mathrm{MPE}=\frac{1}{n^{*}} \sum_{i=1}^{n^{*}} \mathrm{PE}_{i} \quad \text { and } \quad \mathrm{SDPE}_{i}=\sqrt{\frac{1}{n^{*}-1} \sum_{i=1}^{n^{*}}\left(\mathrm{PE}_{i}-\mathrm{MPE}\right)^{2}}
$$

where $n^{*}$ is the sample size of the test set without the outliers. The boxplots in Figures 17 (a) and 17 (b) show the means and standard deviations of the prediction errors for 200 repetitions (i.e. 200 random splits) for the different variable selection methods. The results for the different methods are more or less comparable, with the smallest mean prediction error (as well standard error) for the B-RLARS method, and the largest for the WLAD-LASSO method.

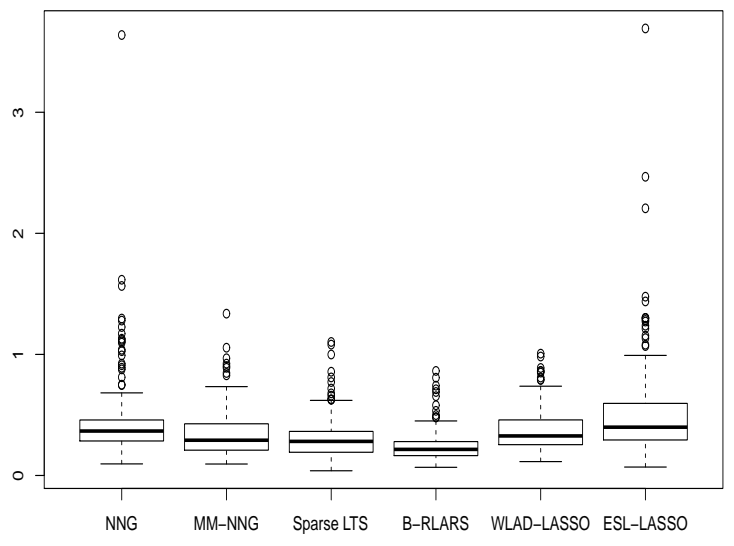

(a)

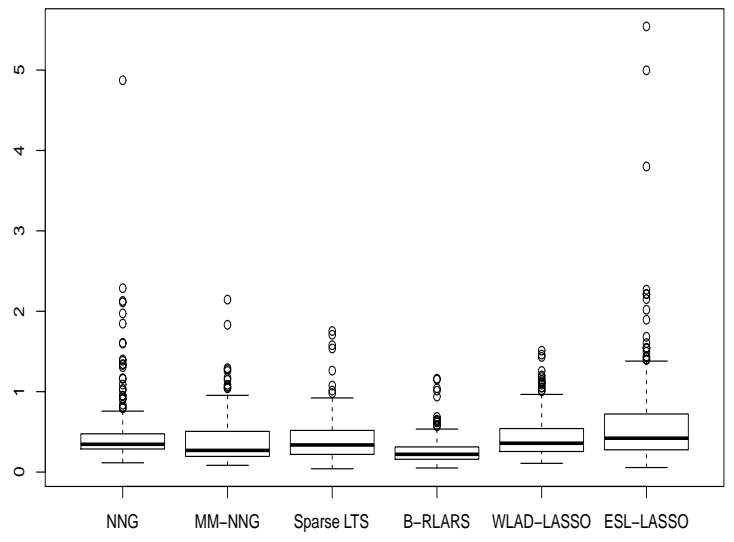

(b)

Figure 18: Boxplots for the means (left) of the prediction errors for "clean" data with extra leverage points.

To see how the performances in prediction is influenced by bad leverage points, the abovedescribed method is carried out again, but now on the "clean" data set of which at random 5 observations are replaced with bad leverage points generated from independent $N(50,1)$ distributions. The training and test sets now have sizes 44 and 10, respectively. The results are shown in Figures 18 (a)\&(b). Also here the best prediction performance is for the B-RLARS method, closely followed by the others, with the weakest performance (in prediction) for the ESL-LASSO method.

\section{Further discussion and conclusion}

The nonnegative garrote is a variable selection method frequently used in practice. Nevertheless, it does not allow for robust estimates. This paper introduces three robust versions of this method 
for linear regression models, namely the M-, LTS, and S-nonnegative garrote. Simulation results illustrated their good performances, if the initial estimator is highly efficient. They also showed that the S-nonnegative garrote performs better than the other two. However, efficiency is an issue with the S-nonnegative garrote. Therefore, we introduced the MM-nonnegative garrote by combining the S- and M-nonnegative garrote. This combination has a higher efficiency than the S-nonnegative garrote, as shown in the simulation study, and often outperforms existing methods such as Sparse LTS and WLAD-LASSO. Close competitors are the B-RLARS and ESL-LASSO methods. Given its good overall performance in the extensive simulation study we recommend the use of the MM-nonnegative garrote method, despite the computational cost.

The authors are currently investigating the theoretical properties of the robust nonnegative garrote methods. Proving consistency in estimation, as well as studying properties of robustness in estimation, such as calculating breakdown points and influence functions, are of interest. These results will appear in a forthcoming manuscript. A first result that has been proven is the consistency of the S-nonnegative garrote method. One of the other findings is that the influence function of a specific robust nonnegative garrote estimator is different for different initial robust estimators, which is to be expected. Apart from studying the influence of outlying observations on the performance of the estimation task, one can also wonder about there impact on the variable selection task. Addressing this question is more than challenging since notions similar to breakdown point, for example, are not available. An attempt to formulate what could possibly be meant by a breakdown point for variable selection can be found in the $\mathrm{PhD}$ thesis of Schumann (2009), but this is far from satisfactory. Moreover, the interesting discussion paper of Davies and Gather (2005) reveals that the concept of breakdown point needs a subtle treatment and is far from easy to extend to new situations, such as breakdown point for principal component analysis. We refer the reader to this paper for many interesting discussions on this and related issues.

Another important issue is appropriate choices of regularization parameters with respect to variable selection. As can also be seen from the simulation results reported on in Sections 3 and 5, accurate estimation and accurate variable selection can be conflicting goals. Indeed, sometimes one gets good performances for the variable selection criteria (MTZ, MFZ, MTP, MFP) in Table 2, and less good performance for the estimation criterion (ER), or visa versa. A crucial question is also how to define an appropriate criterion for selecting the regularization parameters, when both tasks, estimation and variable selection, are aimed at simultaneously. This is a challenging and open research question.

\section{Acknowledgments}

The authors would like to thank an Associate Editor and three additional reviewers for useful comments which led to a considerable improvements of the manuscript. The authors are very grateful to Dr Heping Zhang for providing MATLAB codes for the Exponential Squared Loss LASSO method. This research is supported by the IAP Research Network P7/06 of the Belgian State (Belgian Science Policy), and the project GOA/12/014 of the Research Fund of the KU Leuven.

\section{Appendix}

In this section we provide proofs of Theorems 2.1 and 2.2. 
Proof of Theorem 2.1. The equation of the minimum of (4a) for the M-nonnegative garrote method is given by

$$
\frac{1}{n} \sum_{i=1}^{n} \rho^{\prime}\left(\frac{Y_{i}-\boldsymbol{Z}_{i}^{\prime} \widehat{\mathbf{c}}}{\widehat{\sigma}}\right)\left(\frac{-\boldsymbol{Z}_{i}}{\widehat{\sigma}}\right)+\lambda \mathbf{1}_{p}=0 .
$$

This can also be written as

$$
\boldsymbol{Z}^{\prime} \mathbf{W}_{M}(\widehat{\mathbf{c}}) \boldsymbol{Z} \widehat{\mathbf{c}}-\boldsymbol{Z}^{\prime} \mathbf{W}_{M}(\widehat{\mathbf{c}}) \mathbf{Y}+n \widehat{\sigma}^{2} \lambda \mathbf{1}_{p}=0,
$$

where $\mathbf{W}_{M}(\mathbf{c})=\operatorname{diag}\left(W_{M, i}(\mathbf{c})\right) \in \mathbb{R}^{n \times n}$ with $W_{M, i}(\mathbf{c})=\rho^{\prime}\left(\frac{r_{\mathrm{R}, i}(\mathbf{c})}{\widehat{\sigma}}\right)\left(\frac{\widehat{\sigma}}{r_{\mathrm{R}, i}(\mathbf{c})}\right)$. Thus,

$$
\widehat{\mathbf{c}}=\left(\boldsymbol{Z}^{\prime} \mathbf{W}_{M}(\widehat{\mathbf{c}}) \boldsymbol{Z}\right)^{-1}\left(\boldsymbol{Z}^{\prime} \mathbf{W}_{M}(\widehat{\mathbf{c}}) \mathbf{Y}-n \widehat{\sigma}^{2} \lambda \mathbf{1}_{p}\right),
$$

if $\boldsymbol{Z}^{\prime} \mathbf{W}_{M}(\widehat{\mathbf{c}}) \boldsymbol{Z}$ is invertible. Note that (9) is a critical point of the quadratic function

$$
\frac{1}{2} \mathbf{c}^{\prime} \boldsymbol{Z}^{\prime} \mathbf{W}_{M}(\widehat{\mathbf{c}}) \boldsymbol{Z} \mathbf{c}-\left(\boldsymbol{Z}^{\prime} \mathbf{W}_{M}(\widehat{\mathbf{c}}) \mathbf{Y}-n \widehat{\sigma}^{2} \lambda \mathbf{1}_{p}\right)^{\prime} \mathbf{c} .
$$

Therefore, if the constraint (4b) is also considered, the optimization problem (4a)\&(4b) for the M-nonnegative garrote method can be written as

$$
\left\{\begin{array}{l}
\widehat{\mathbf{c}}=\underset{\mathbf{c}}{\operatorname{argmin}}\left\{\frac{1}{2} \mathbf{c}^{\prime} \boldsymbol{Z}^{\prime} \mathbf{W}_{M}\left(\widehat{\mathbf{c}}^{0}\right) \boldsymbol{Z} \mathbf{c}-\left(\boldsymbol{Z}^{\prime} \mathbf{W}_{M}\left(\widehat{\mathbf{c}}^{0}\right) \mathbf{Y}-n \widehat{\sigma}^{2} \lambda \mathbf{1}_{p}\right)^{\prime} \mathbf{c}\right\} \\
\text { s.t. } c_{j} \geq 0(j=1, \ldots, p),
\end{array}\right.
$$

with $\widehat{\mathbf{c}}^{0}$ the current value of $\mathbf{c}$ in the iterative procedure.

Proof of Theorem 2.2. The equation for the minimum of (4a) for the S-nonnegative garrote method is given by

$$
\nabla \widehat{\sigma}\left(\mathbf{r}_{\mathrm{R}}(\widehat{\mathbf{c}})\right)+\lambda \mathbf{1}_{p}=0
$$

where $\nabla \widehat{\sigma}\left(\mathbf{r}_{\mathrm{R}}(\mathbf{c})\right)=\frac{\partial \widehat{\sigma}\left(\mathbf{r}_{\mathrm{R}}(\mathbf{c})\right)}{\partial \mathbf{c}}$. To compute $\nabla \widehat{\sigma}\left(\mathbf{r}_{\mathrm{R}}(\mathbf{c})\right)$, the derivative of equation (5) with respect to $\mathbf{c}$ is taken. For $\widehat{\sigma}\left(\mathbf{r}_{\mathrm{R}}(\mathbf{c})\right) \neq 0$, we obtain

$$
\sum_{i=1}^{n} \rho^{\prime}\left(\frac{r_{\mathrm{R}, i}(\mathbf{c})}{\widehat{\sigma}\left(\mathbf{r}_{\mathrm{R}}(\mathbf{c})\right)}\right)\left(\frac{-\boldsymbol{Z}_{i} \widehat{\sigma}\left(\mathbf{r}_{\mathrm{R}}(\mathbf{c})\right)-r_{\mathrm{R}, i}(\mathbf{c}) \nabla \widehat{\sigma}\left(\mathbf{r}_{\mathrm{R}}(\mathbf{c})\right)}{\widehat{\sigma}^{2}\left(\mathbf{r}_{\mathrm{R}}(\mathbf{c})\right)}\right)=0
$$

or

$$
-\sum_{i=1}^{n} \rho^{\prime}\left(\frac{r_{\mathrm{R}, i}(\mathbf{c})}{\widehat{\sigma}\left(\mathbf{r}_{\mathrm{R}}(\mathbf{c})\right)}\right) \frac{\boldsymbol{Z}_{i} \widehat{\sigma}\left(\mathbf{r}_{\mathrm{R}}(\mathbf{c})\right)}{\widehat{\sigma}^{2}\left(\mathbf{r}_{\mathrm{R}}(\mathbf{c})\right)}=\sum_{i=1}^{n} \rho^{\prime}\left(\frac{r_{\mathrm{R}, i}(\mathbf{c})}{\widehat{\sigma}\left(\mathbf{r}_{\mathrm{R}}(\mathbf{c})\right)}\right) \frac{r_{\mathrm{R}, i}(\mathbf{c}) \nabla \widehat{\sigma}\left(\mathbf{r}_{\mathrm{R}}(\mathbf{c})\right)}{\widehat{\sigma}^{2}\left(\mathbf{r}_{\mathrm{R}}(\mathbf{c})\right)} .
$$

The derivative of the M-scale $\widehat{\sigma}\left(\mathbf{r}_{\mathrm{R}}(\mathbf{c})\right)$ with respect to $\mathbf{c}$ is therefore given by,

$$
\nabla \widehat{\sigma}\left(\mathbf{r}_{\mathrm{R}}(\mathbf{c})\right)=\frac{-\sum_{i=1}^{n} \rho^{\prime}\left(\frac{r_{\mathrm{R}, i}(\mathbf{c})}{\widehat{\sigma}\left(\mathbf{r}_{\mathrm{R}}(\mathbf{c})\right)}\right) \boldsymbol{Z}_{i}}{\sum_{i=1}^{n} \rho^{\prime}\left(\frac{r_{\mathrm{R}, i}(\mathbf{c})}{\widehat{\sigma}\left(\mathbf{r}_{\mathrm{R}}(\mathbf{c})\right)}\right) \frac{r_{\mathrm{R}, i}(\mathbf{c})}{\widehat{\sigma}\left(\mathbf{r}_{\mathrm{R}}(\mathbf{c})\right)}}=-\frac{\widehat{\sigma}\left(\mathbf{r}_{\mathrm{R}}(\mathbf{c})\right) \boldsymbol{Z}^{\prime} \mathbf{W}_{S}(\mathbf{c}) \mathbf{r}_{\mathrm{R}}(\mathbf{c})}{\mathbf{r}_{\mathrm{R}}^{\prime}(\mathbf{c}) \mathbf{W}_{S}(\mathbf{c}) \mathbf{r}_{\mathrm{R}}(\mathbf{c})}
$$


where $\mathbf{W}_{S}(\mathbf{c})=\operatorname{diag}\left(W_{S, i}(\mathbf{c})\right) \in \mathbb{R}^{n \times n}$ with $W_{S, i}(\mathbf{c})=\rho^{\prime}\left(\frac{r_{\mathrm{R}, i}(\mathbf{c})}{\widehat{\sigma}\left(\mathbf{r}_{\mathrm{R}}(\mathbf{c})\right)}\right)\left(\frac{\widehat{\sigma}\left(\mathbf{r}_{\mathrm{R}}(\mathbf{c})\right)}{r_{\mathrm{R}, i}(\mathbf{c})}\right)$. Plugging (11) into equation (10) gives

$$
-\frac{\widehat{\sigma}\left(\mathbf{r}_{\mathrm{R}}(\widehat{\mathbf{c}})\right) \boldsymbol{Z}^{\prime} \mathbf{W}_{S}(\widehat{\mathbf{c}}) \mathbf{r}_{\mathrm{R}}(\widehat{\mathbf{c}})}{\mathbf{r}_{\mathrm{R}}^{\prime}(\widehat{\mathbf{c}}) \mathbf{W}_{S}(\widehat{\mathbf{c}}) \mathbf{r}_{\mathrm{R}}(\widehat{\mathbf{c}})}+\lambda \mathbf{1}_{p}=0
$$

Denote with $\omega_{S}(\mathbf{c})=\frac{\widehat{\sigma}\left(\mathbf{r}_{\mathrm{R}}(\mathbf{c})\right)}{\mathbf{r}_{\mathrm{R}}^{\prime}(\mathbf{c}) \mathbf{W}_{S}(\mathbf{c}) \mathbf{r}_{\mathrm{R}}(\mathbf{c})}$, then it follows that

$$
-\omega_{S}(\widehat{\mathbf{c}}) \boldsymbol{Z}^{\prime} \mathbf{W}_{S}(\widehat{\mathbf{c}})(\mathbf{Y}-\boldsymbol{Z} \widehat{\mathbf{c}})+\lambda \mathbf{1}_{p}=0,
$$

or

$$
\widehat{\mathbf{c}}=\left(\boldsymbol{Z}^{\prime} \mathbf{W}_{S}(\widehat{\mathbf{c}}) \boldsymbol{Z}\right)^{-1}\left(\boldsymbol{Z}^{\prime} \mathbf{W}_{S}(\widehat{\mathbf{c}}) \mathbf{Y}-\frac{\lambda}{\omega_{S}(\widehat{\mathbf{c}})} \mathbf{1}_{p}\right),
$$

if $\boldsymbol{Z}^{\prime} \mathbf{W}_{S}(\widehat{\mathbf{c}}) \boldsymbol{Z}$ is invertible. The generalized inverse of $\boldsymbol{Z}^{\prime} \mathbf{W}_{S}(\widehat{\mathbf{c}}) \boldsymbol{Z}$ can be used, if at least one of the robust initial estimates $\widehat{\beta}_{j}^{\mathrm{R}}, j=1, \ldots, p$, is equal to zero, since the matrix $\boldsymbol{Z}^{\prime} \mathbf{W}_{S}(\widehat{\mathbf{c}}) \boldsymbol{Z}$ is then singular. Remark that (12) is a critical point of the quadratic function

$$
\frac{1}{2} \mathbf{c}^{\prime} \boldsymbol{Z}^{\prime} \mathbf{W}_{S}(\widehat{\mathbf{c}}) \boldsymbol{Z} \mathbf{c}-\left(\boldsymbol{Z}^{\prime} \mathbf{W}_{S}(\widehat{\mathbf{c}}) \mathbf{Y}-\frac{\lambda}{\omega_{S}(\widehat{\mathbf{c}})} \mathbf{1}_{p}\right)^{\prime} \mathbf{c} .
$$

Hence, if constraint (4b) is also taken into account, the optimization problem (4a)\&(4b) for the S-nonnegative garrote method can be written as a quadratic programming problem

$$
\left\{\begin{array}{l}
\widehat{\mathbf{c}}=\underset{\mathbf{c}}{\operatorname{argmin}}\left\{\frac{1}{2} \mathbf{c}^{\prime} \boldsymbol{Z}^{\prime} \mathbf{W}_{S}\left(\widehat{\mathbf{c}}^{0}\right) \boldsymbol{Z} \mathbf{c}-\left(\boldsymbol{Z}^{\prime} \mathbf{W}_{S}\left(\widehat{\mathbf{c}}^{0}\right) \mathbf{Y}-\frac{\lambda}{\omega_{S}(\widehat{\mathbf{c}})} \mathbf{1}_{p}\right)^{\prime} \mathbf{c}\right\} \\
\text { s.t. } c_{j} \geq 0(j=1, \ldots, p),
\end{array}\right.
$$

with $\widehat{\mathbf{c}}^{0}$ the current value of $\mathbf{c}$ in the iterative procedure.

\section{References}

Agostinelli, C. and Salibian-Barrera, M. (2010). Robust model selection with lars based on s-estimators. In Y Lechevallier and G Saporta, Proceedings of COMPSTAT 2010, 19th International Conference on Computational Statistics, pages 69-78. Physica-Verlag, Berlin, Paris.

Alfons, A., Croux, C., and Gelper, S. (2013). Sparse least trimmed squares regression for analyzing high-dimensional large data sets. The Annals of Applied Statistics, 7(1):226-248.

Antoniadis, A., Gijbels, I., and Verhasselt, A. (2012a). Variable selection in additive models using $P$-splines. Technometrics, 54(4):425-438.

Antoniadis, A., Gijbels, I., and Verhasselt, A. (2012b). Variable selection in varying-coefficient models using P-splines. Journal of Computational and Graphical Statistics, 21(3):638-661.

Arslan, O. (2012). Weighted LAD-LASSO method for robust parameter estimation and variable selection in regression. Computational Statistics \& Data Analysis, 56(6):1952-1965. 
Belge, M., Kilmer, M. E., and Miller, E. L. (2002). Efficient determination of multiple regularization parameters in a generalized L-curve framework. Inverse Problems, 18(4):1161-1183.

Breiman, L. (1995). Better subset regression using the nonnegative garrote. Technometrics, 37(4):373-384.

Davies, P. L. and Gather, U. (2005). Breakdown and groups. Ann. Statist., 33(3):977-1035. With discussions and a rejoinder by the authors.

Efron, B., Hastie, T., Johnstone, I., and Tibshirani, R. (2004). Least angle regression. The Annals of Statistics, 32(2):407-499. With discussion, and a rejoinder by the authors.

Fan, J. and Li, R. (2001). Variable selection via nonconcave penalized likelihood and its oracle properties. Journal of the American Statistical Association, 96(456):1348-1360.

Frank, I. E. and Friedman, J. H. (1993). A statistical view of some chemometrics regression tools. Technometrics, 35(2):109-135.

Fu, W. J. (1998). Penalized regressions: the bridge versus the lasso. Journal of Computational and Graphical Statistics, 7(3):397-416.

Heritier, S., Cantoni, E., Copt, S., and Victoria-Feser, M.-P. (2009). Robust methods in biostatistics. Wiley Series in Probability and Statistics. John Wiley \& Sons, Ltd., Chichester.

Huber, P. J. (1973). Robust regression: asymptotics, conjectures and Monte Carlo. The Annals of Statistics, 1:799-821.

Khan, J. A., Van Aelst, S., and Zamar, R. H. (2007). Robust linear model selection based on least angle regression. Journal of the American Statistical Association, 102(480):1289-1299.

Khan, J. A., Van Aelst, S., and Zamar, R. H. (2010). Fast robust estimation of prediction error based on resampling. Computational Statistics \& Data Analysis, 54(12):3121-3130.

Koller, M. (2012). Nonsingular subsampling for s-estimators with categorical predictors. ArXiv e-prints, arXiv:1208.5595v1.

Maronna, R. A., Martin, R. D., and Yohai, V. J. (2006). Robust statistics. Wiley Series in Probability and Statistics. John Wiley \& Sons, Ltd., Chichester. Theory and methods.

Medina, M. A. and Ronchetti, E. (2014). Robust and consistent variable selection for generalized linear and additive models. Technical report, University of Geneva, Switserland. http://archive-ouverte.unige.ch/unige:36961.

Owen, A. B. (2006). A robust hybrid of lasso and ridge regression. Technical report, Department of Statistics, Stanford University.

Rousseeuw, P. and Yohai, V. (1984). Robust regression by means of S-estimators. In Robust and nonlinear time series analysis (Heidelberg, 1983), volume 26 of Lecture Notes in Statist., pages 256-272. Springer, New York.

Rousseeuw, P. J. (1984). Least median of squares regression. Journal of the American Statistical Association, 79(388):871-880.

Rousseeuw, P. J. and Driessen, K. V. (2006). Computing LTS regression for large data sets. Data Mining and Knowledge Discovery, 12(1):29-45. 
Salibian-Barrera, M. and Yohai, V. J. (2006). A fast algorithm for S-regression estimates. Journal of Computational and Graphical Statistics, 15(2):414-427.

Salibian-Barrera, M. and Zamar, R. H. (2002). Bootstrapping robust estimates of regression. Ann. Statist., 30(2):556-582.

Schumann, D. (2009). Robust variable selection. ProQuest LLC, Ann Arbor, MI. Thesis (Ph.D.)-North Carolina State University.

Tibshirani, R. (1996). Regression shrinkage and selection via the lasso. Journal of the Royal Statistical Society. Series B. Methodological, 58(1):267-288.

Wang, H., Li, G., and Jiang, G. (2007). Robust regression shrinkage and consistent variable selection through the LAD-Lasso. Journal of Business 83 Economic Statistics, 25(3):347-355.

Wang, L. and Li, R. (2009). Weighted Wilcoxon-type smoothly clipped absolute deviation method. Biometrics. Journal of the International Biometric Society, 65(2):564-571.

Wang, X., Jiang, Y., Huang, M., and Zhang, H. (2013). Robust variable selection with exponential squared loss. Journal of the American Statistical Association, 108(502):632-643.

Yohai, V. J. (1987). High breakdown-point and high efficiency robust estimates for regression. The Annals of Statistics, 15(2):642-656.

Yohai, V. J. and Zamar, R. H. (1988). High breakdown-point estimates of regression by means of the minimization of an efficient scale. Journal of the American Statistical Association, 83(402):406-413.

Yuan, M. and Lin, Y. (2007). On the non-negative garrote estimator. Journal of the Royal Statistical Society. Series B. Statistical Methodology, 69(2):143-161. 


\title{
Supplemental material to the paper
}

\section{Robust nonnegative garrote variable selection in linear regression,}

\author{
by I. Gijbels and I. Vrinssen
}

\section{Additional simulation results for an increased contamination level}

Here we repeat the simulation study of Section 5, but now with a contamination level of $20 \%$ outliers instead of $10 \%$. The results for contamination schemes $2-5$ are shown in Figures 19-24. From these figures it is clear that, as expected, all methods perform less well. It is of interest to note that the ESL-LASSO method seems to suffer slightly less from the increased contamination level, and its influence is most noted in an increase in estimation error.

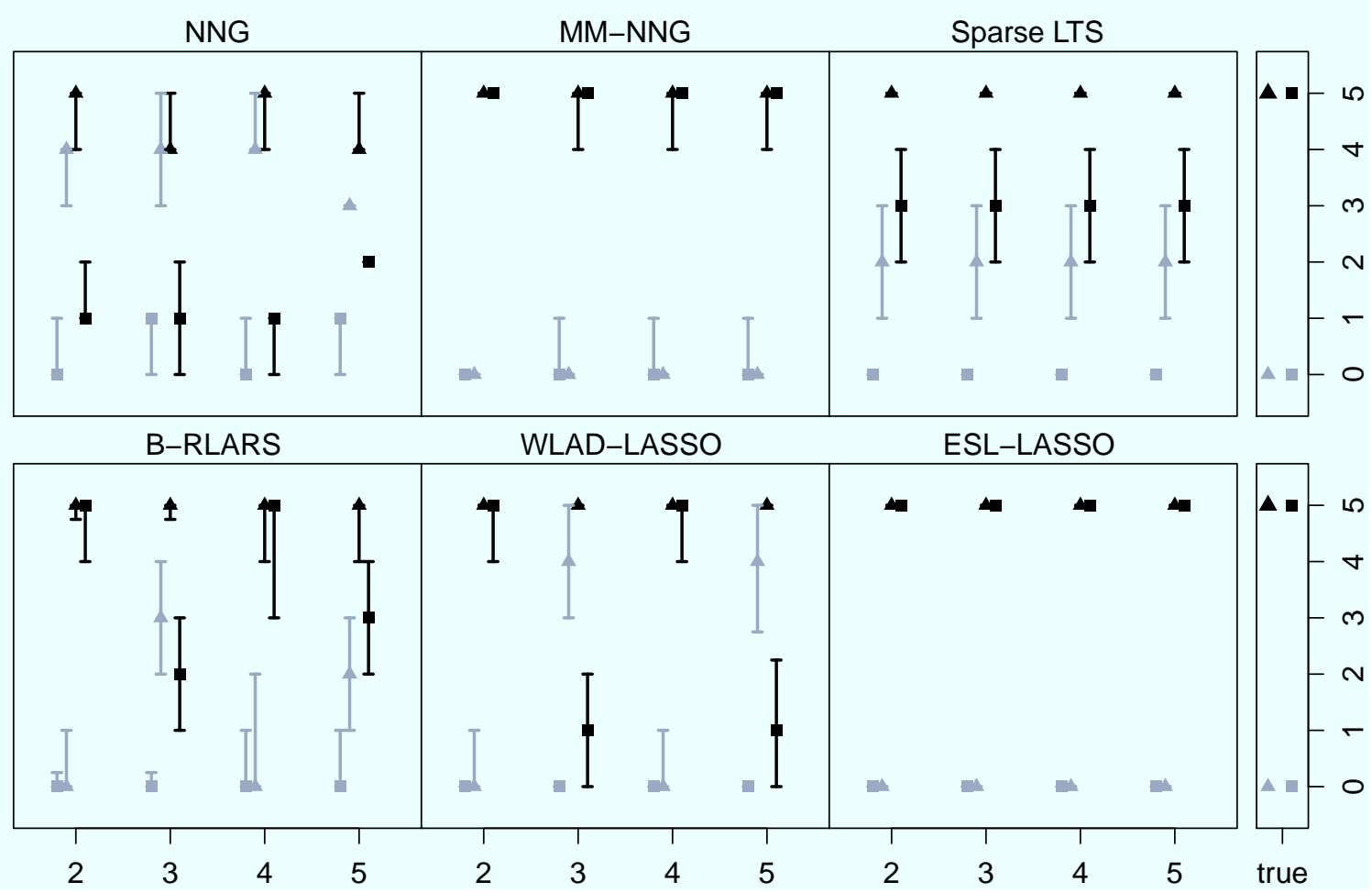

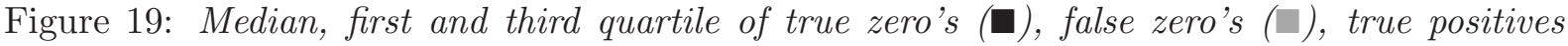
$(\Delta)$ and false positives ( $\Delta$ ) for simulation example 1, with contamination level 20\%. The optimal values are $M T Z=5, M F Z=0, M T P=5$ and $M F P=0$ and they are plotted in the side bar. 


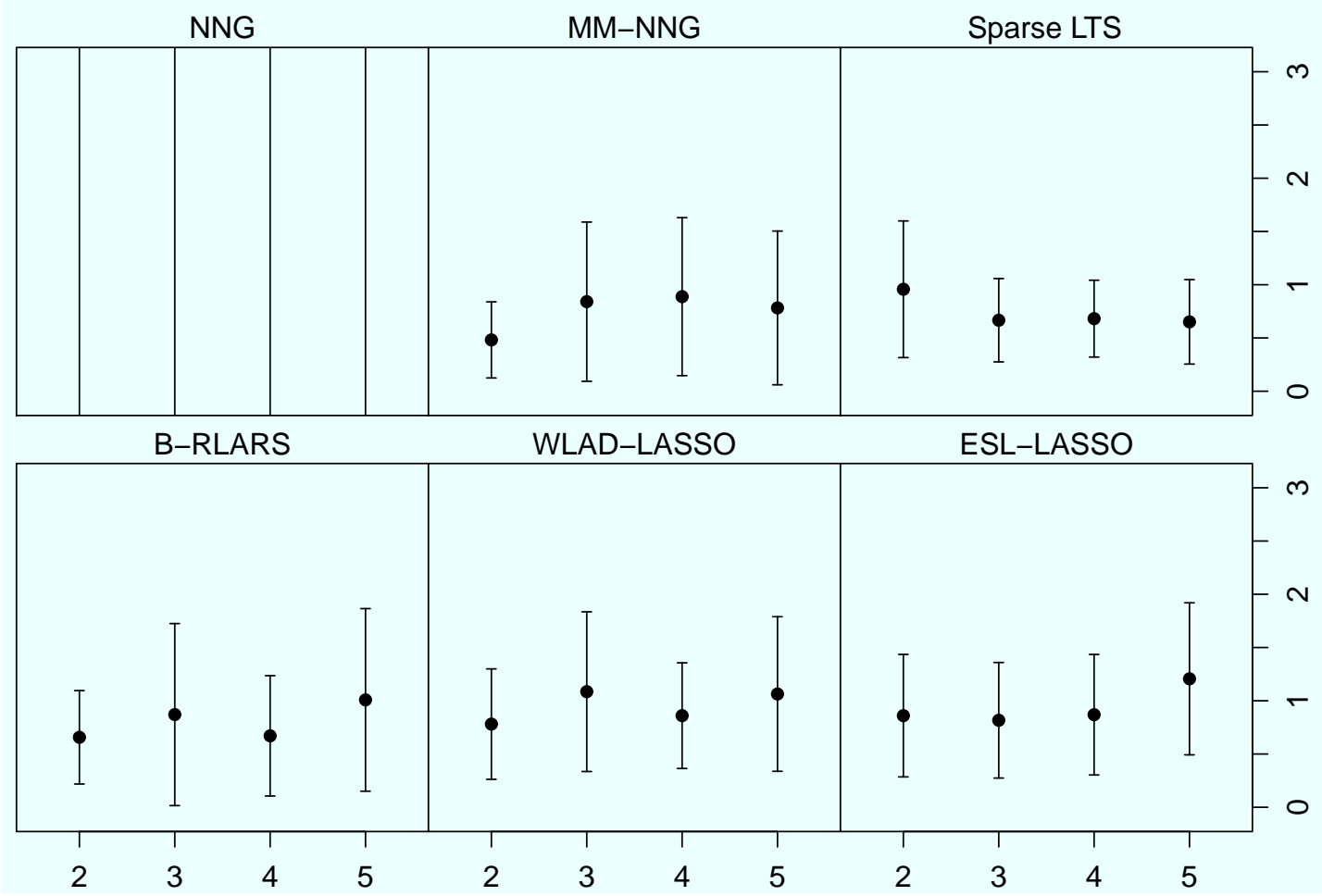

Figure 20: Mean and standard deviation of the estimation error for simulation example 1, with contamination level $20 \%$.

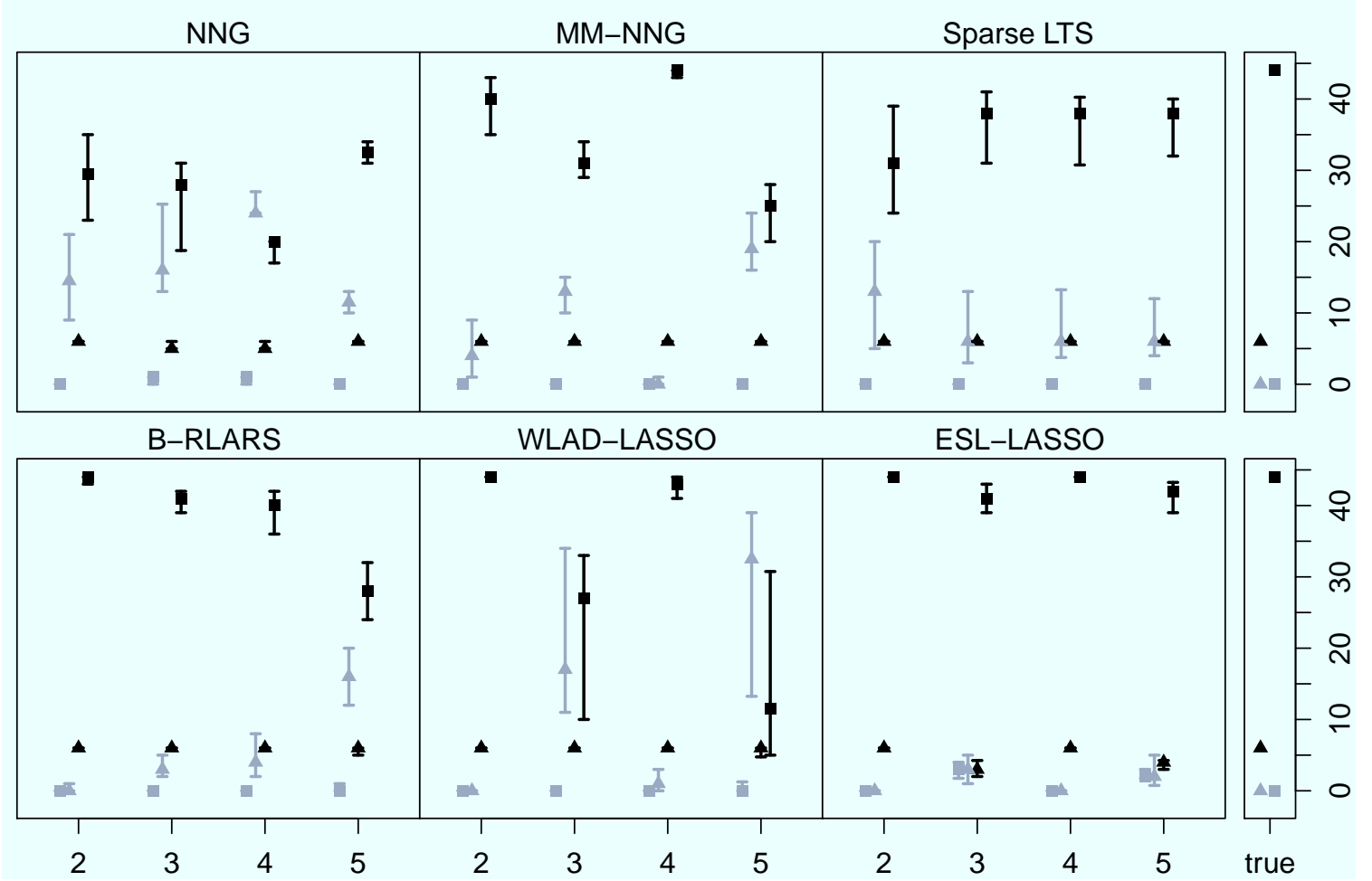

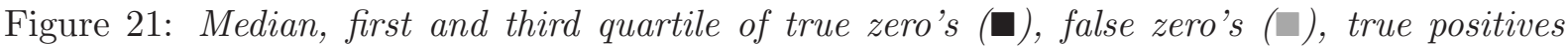
$(\Delta)$ and false positives ( $\Delta$ ) for simulation example 2, with contamination level 20\%. The optimal values are $M T Z=44, M F Z=0, M T P=6$ and $M F P=0$ and they are plotted in the side bar. 


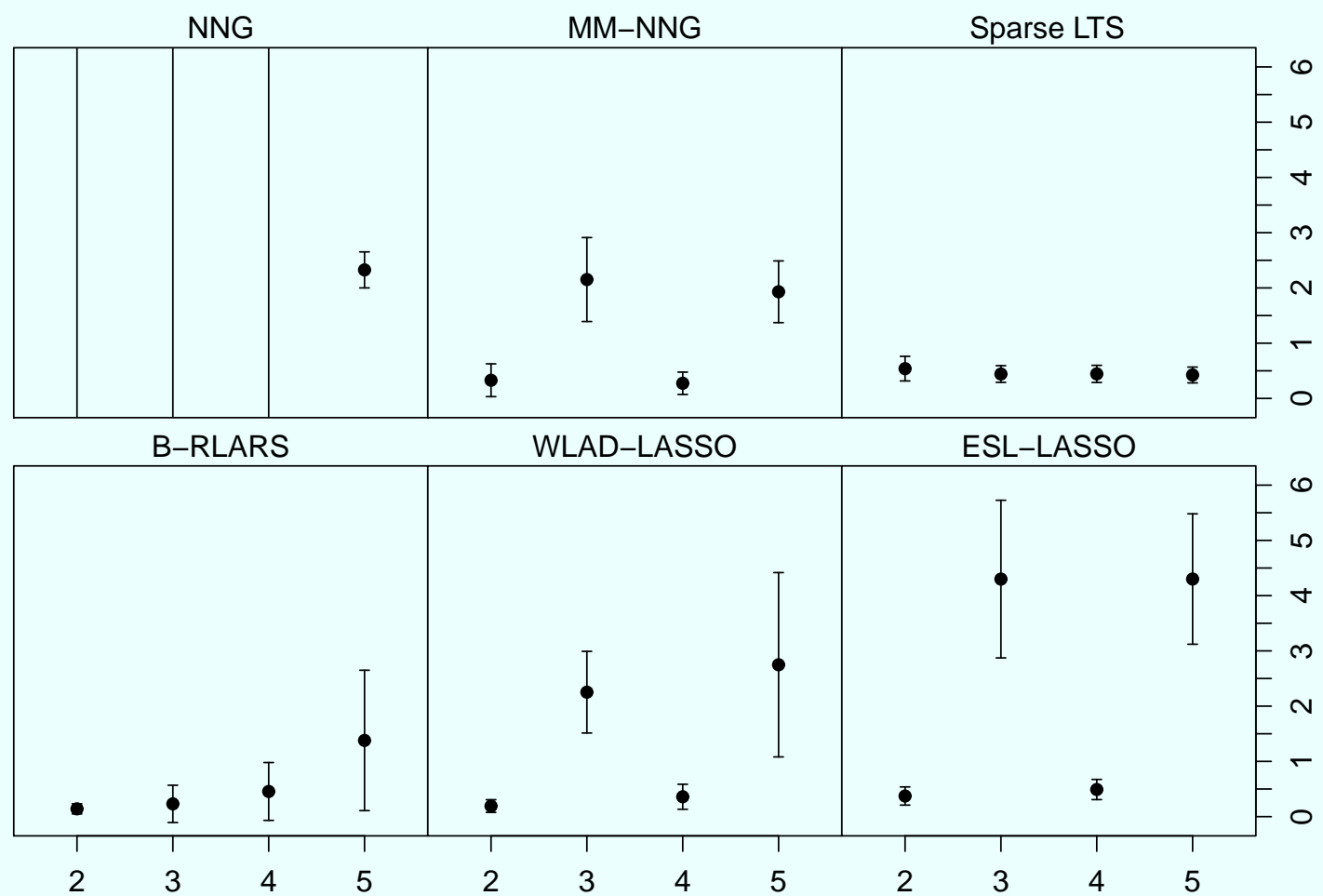

Figure 22: Mean and standard deviation of the estimation error for simulation example 2, with contamination level $20 \%$.

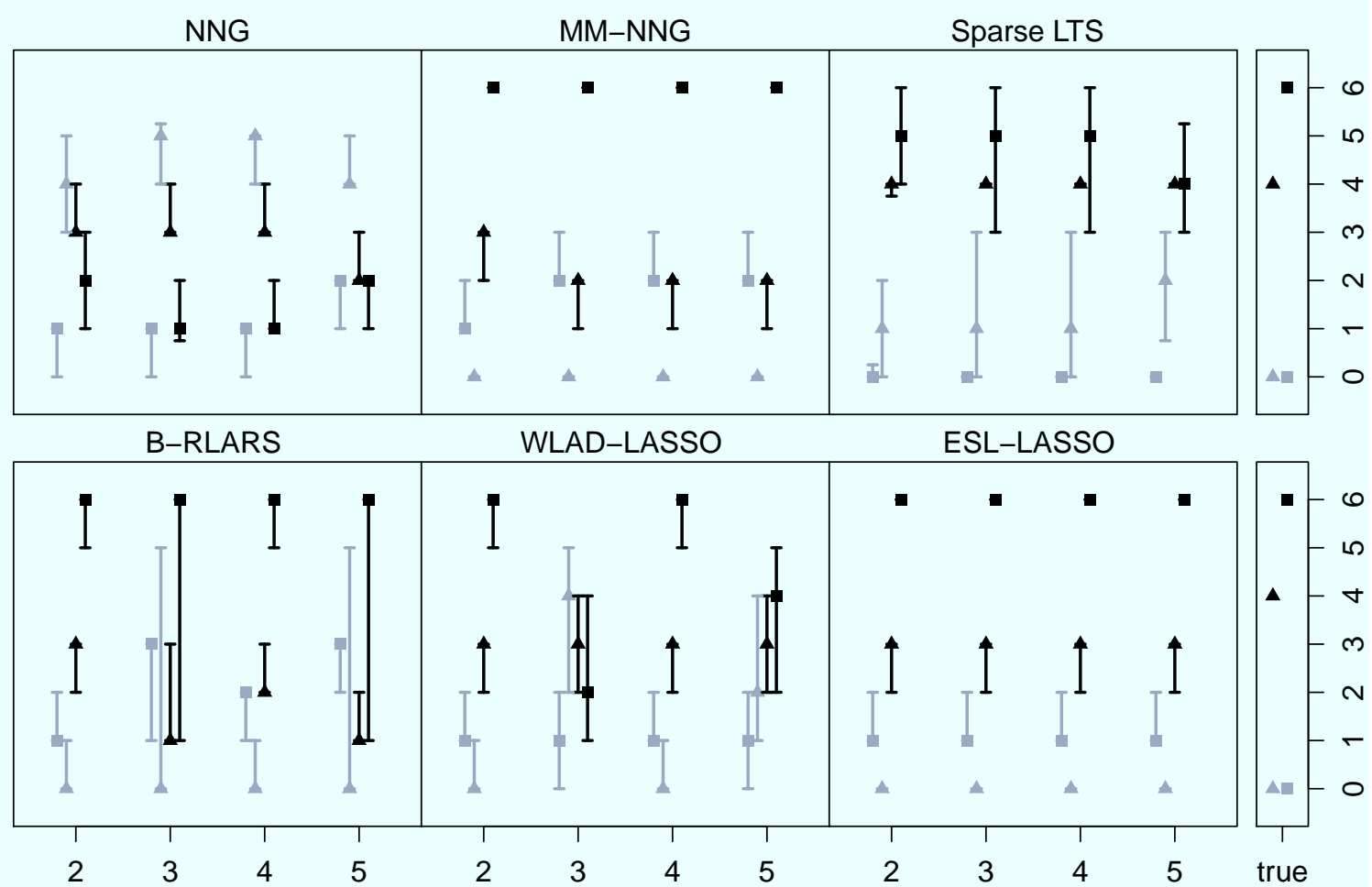

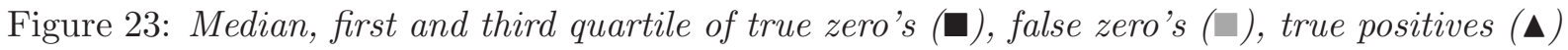
and false positives ( $\Delta$ ) for simulation example 3, with contamination level 20\%. The optimal values are $M T Z=6, M F Z=0, M T P=4$ and $M F P=0$ and they are plotted in the side bar. 


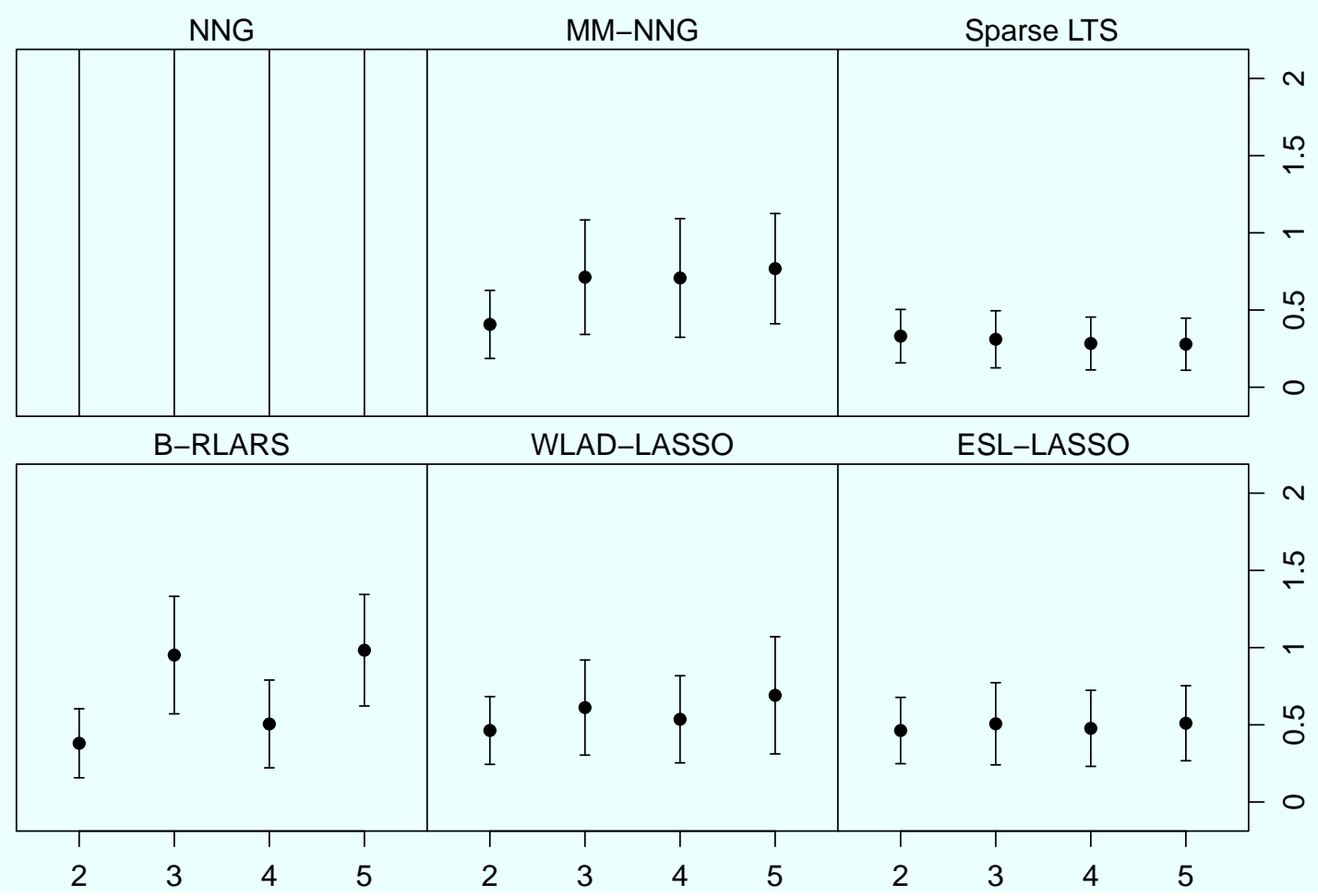

Figure 24: Mean and standard deviation of the estimation error for simulation example 3, with contamination level $20 \%$. 